\title{
The Study of Key Tongue Features for Prediabetes Risk and Diabetes Risk using Principal Component Analysis and Logistic Regression
}

\section{Jun Li ( $\square$ ljzy05@126.com )}

Shanghai University of Traditional Chinese Medicine https://orcid.org/0000-0001-5624-2146

Jiacai Li

Shanghai University of Traditional Chinese Medicine

\section{Xiaojuan Hu}

Shanghai University of Traditional Chinese Medicine

\section{Liping Tu}

Shanghai University of Traditional Chinese Medicine

\section{Yulin Shi}

Shanghai University of Traditional Chinese Medicine

\section{Zijuan Bi}

Shanghai University of Traditional Chinese Medicine

\section{Changle Zhou}

Shanghai University of Traditional Chinese Medicine

\section{Ji Cui}

Shanghai University of Traditional Chinese Medicine Jiatuo Xu

Shanghai University of Traditional Chinese Medicine

\section{Research}

Keywords: Tongue image, Tongue diagnosis, Prediabetes, Diabetes, PCA, Logistic regression

Posted Date: June 3rd, 2021

DOl: https://doi.org/10.21203/rs.3.rs-557559/v1

License: (c) (1) This work is licensed under a Creative Commons Attribution 4.0 International License. Read Full License 


\title{
The study of key tongue features for prediabetes risk and diabetes risk using principal component analysis and logistic regression
}

\author{
Jun $\mathrm{Li}^{1 \dagger}$, Jiacai $\mathrm{Li}^{1}$, Xiaojuan $\mathrm{Hu}^{2}$, Liping $\mathrm{Tu}^{1}$, Yulin Shi ${ }^{1}$, Zijuan $\mathrm{Bi}^{1}$, Changle Zhou ${ }^{3}$, Ji Cui ${ }^{1^{*}}$, Jiatuo $\mathrm{Xu}^{1^{*}}$
}

1 School of Basic Medicine, Shanghai University of Traditional Chinese Medicine, Shanghai, China

2 Shanghai Collaborative Innovation Center of Health Service in Traditional Chinese Medicine, Shanghai University of Traditional Chinese Medicine, Shanghai, China

3 Cognitive Science Department, Xiamen University, Xiamen, China

${ }^{*}$ Co-corresponding author:

Dr. Ji Cui

Address: School of Basic Medicine, Shanghai University of Traditional Chinese Medicine, Cailun Road, Shanghai, China

Email: maatcui@163.com

${ }^{*}$ Corresponding Author:

Prof. Jiatuo Xu

Address: School of Basic Medicine, Shanghai University of Traditional Chinese Medicine, Cailun Road, Shanghai, China Phone: 862151322432

Email: xjt@fudan.edu.cn

\section{Abstract}

Background: Carrying out routine diabetes risk assessment and prediction is conducive to controlling the incidence of diabetes. Previous studies have confirmed that tongue features can reflect changes in glucose metabolism. We want to find the key tongue features related to glucose metabolism for early warning of prediabetes and diabetes. This will benefit the prevention and control of diabetes.

Methods: We investigated 719 subjects in Shuguang Hospital affiliated with Shanghai University of TCM in Shanghai, China, during the August 2018 - December 2019 period. We used PCA to reconstruct 25 new features on the basis of the original features, and used logistic regression to analyze these new features. Based on the factor loading method, we associate the new features with the original features. Finally, we determined the key tongue features and change patterns related to prediabetes risk and diabetes risk.

Results: For the prediabetes, TB-ASM, TC-ASM and TB-Cr are protective factors. TB-CON, TC-CON, TBMEAN, TC-MEAN, TB-ENT and TC-ENT are risk factors. For the diabetes, TB-a, TB-S, TB-Cr, TB-b and TC-b are protective factors, and Per-all and TB-Cb are risk factors. 
Conclusion: The PCA eliminates the redundancy of original features and refines the original features. The PCA combined with logistic regression found the key tongue features reflecting glucose metabolism, and clarified the significance of the changes in these tongue features in the prediabetes and diabetes population. This study provides a research foundation for using tongue features to implement early warning of diabetes risk.

Keywords: Tongue image, Tongue diagnosis, Prediabetes, Diabetes, PCA, Logistic regression.

\section{Introduction}

Tongue diagnosis is a rapid, objective, simple, noninvasive and valuable method that provides access to physiology and pathology information for TCM doctor. The doctor observes the color, texture and moisture of the tongue body and tongue coating to identify the health status of an individual. At present, with the development of standardized tongue diagnosis equipment, objective tongue information continues to accumulate. Researchers use tongue diagnosis equipment to collect and analyze the subjects' tongue images, calculate and extract tongue features.

According to $\mathrm{WHO}$, the prediabetes is associated with a high risk for developing diabetes. In addition, prediabetes is related to early atherosclerosis, subclinical inflammation, and cardiovascular disease (CVD). The occurrence of prediabetes is due to the joint action of insulin resistance and $\beta$-cell dysfunction ${ }^{[1,2]}$. WHO has set the prevention of T2DM as a priority goal. At the same time, the 2018 Berlin Declaration called on the world to take early measures to prevent T2DM. Individuals at high risk of diabetes should actively implement lifestyle modification ${ }^{[3]}$. Many diabetic patients were missed due to hidden onset

${ }^{[4]}$. Delay in diagnosis and treatment not only affects the patient's prognosis, but also incurs high medical costs ${ }^{[5,6]}$. Therefore, it is necessary to establish a routine diabetes risk screening mechanism. However, Routine blood glucose monitoring is an invasive examination and will incur a certain cost. Therefore, it is necessary to establish a low-cost, convenient, non-invasive, and accurate diabetes risk screening system. For example, The American Diabetes Association (ADA) recommends an noninvasive assessment tool to assess diabetes risk ${ }^{[7]}$.

Our previous studies have confirmed that tongue features has a potential role in predicting risks of diabetes ${ }^{[8]}$. However, among the many tongue features, which tongue features are more important in the early warning of hyperglycemia and still need to be explored. Using PCA and logistic regression analysis, we hope to reconstruct new features on the basis of the original features to solve the collinear and redundancy of the original features, and to find features that can accurately distinguish between normal and hyperglycemia, especially find the key tongue features for risk warning of prediabetes and diabetes. This will benefit the prevention and treatment of diabetes.

\section{Methods}

\section{Subjects}

In our study, we investigated 719 subjects in Shuguang Hospital affiliated with Shanghai University of TCM in Shanghai, China during the August 2018 - December 2019 period. Subjects were divided into normal case $(3.9 \mathrm{mmol} / \mathrm{L}<$ fasting blood glucose $(\mathrm{FPG})<6.1 \mathrm{mmol} / \mathrm{L}$ and $\mathrm{HbAlc}<5.7 \%$ ), and those with prediabetes (FPG 6.1-6.9mmol/L or HbAlc 5.7-6.4\%), and 
and those with diabetes (FPG $\geqslant 7.0 \mathrm{mmol} / \mathrm{L}$ or $\mathrm{HbAlc} \geqslant 6.5 \%$ ). We confirm that all experiments were performed in accordance with relevant guidelines and regulations. All subjects signed an informed consent form. The study was approved by the IRB of Shuguang Hospital affiliated with Shanghai University of TCM.

\section{Collection of clinical data}

We collected basic information, blood samples and tongue images of subjects on the same day. Basic information included: Gender, Age, Height, Weight, Systolic blood pressure (SBP), Diastolic blood pressure (DBP).

Laboratory results included: 1) blood routine tests including white blood cell (WBC) count, neutrophils (NE\#), lymphocytes (LY\#), monocytes (MO\#), eosinophils (EO\#), basophils (BA\#), red blood cell (RBC) count, red cell distribution width coefficient of variation (RDW-CV), hematocrit (HCT), mean corpuscular volume (MCV), hemoglobin (HGB), Mean corpuscular hemoglobin $(\mathrm{MCH})$, mean corpuscular hemoglobin concentration (MCHC), platelet (PLT) count, platelet distribution width (PDW), plateletcrit (PCT), mean platelet volume (MPV). 2) blood chemistry tests including: Total cholesterol (TCHO), triglycerides (TG), low-density lipoprotein (LDL), high-density lipoprotein (HDL), uric acid (UA), total protein (TP), globulin (GLB), albumin (ALB), blood urea(UREA) nitrogen, creatinine (CREA), estimated glomerular filtration rate (eGFR), total bile acids (TBA), total bilirubin (TBIL), direct bilirubin (DBIL), indirect bilirubin (IBIL), alkaline phosphatase (ALP), alanine aminotransferase (ALT), gamma-glutamyl transpeptidase (GGT), aspartate transaminase (AST) (Table 1).

\section{Collection and analysis of tongue features}

Tongue image acquisition equipment (type TFDA-1 tongue diagnosis instrument) (Fig. 1) and analysis software (Tongue diagnosis analysis system, TDAS) were designed and developed by the intelligent diagnosis technology research team in Shanghai University of TCM ${ }^{[9]}$. TFDA-1 is equipped with a high-definition camera and standard light source to ensure a stable shooting environment. The tongue images were recorded as JPEG files (5568 x 3712 pixels).

Based on the color image segmentation method ${ }^{[10]}$, TDAS first implemented tongue segmentation. According to the difference between the pixel values of the tongue coating and the tongue body, the tongue coating was separated from the tongue body and the color features of both in the RGB, Lab, HIS, YCrCb color space were calculated. According to the difference statistical method ${ }^{[11]}$, TDAS automatically calculates the texture features of tongue body and tongue coating. The principle of tongue coating ratio feature calculation was: tongue coating area calculated based on pixel value/full tongue area (Per-all), tongue coating area calculated based on pixel value/tongue coating area calculated based on pixel position (Perpart). 


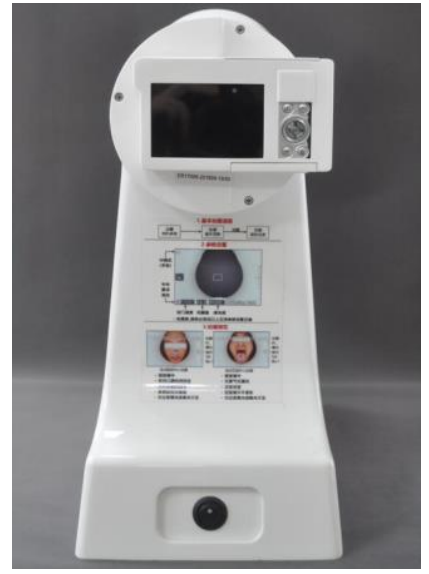

(a) Rear view

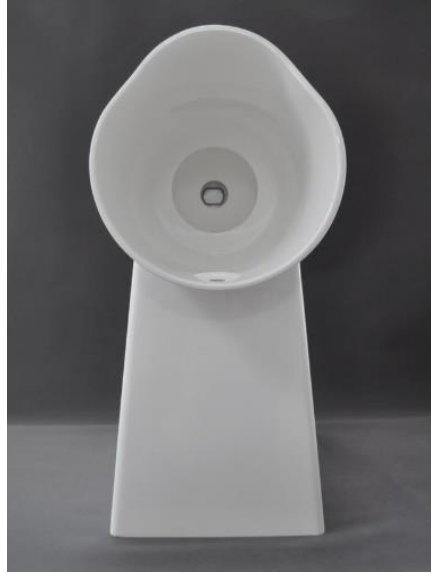

(b) Front view

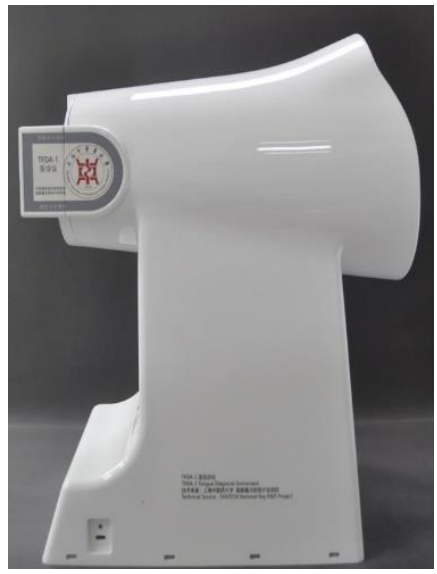

(c) Side view

Fig.1 Type TFDA-1 tongue diagnosis instrument

\section{Statistical analysis and machine learning modeling}

The measurement data presented as $\bar{X} \pm S D$ and median (interquartile range). For measurement data that conformed to a normal distribution and homogeneity of variance, the independent sample t-test was used, and for measurement data that did not conform to a normal distribution, the Mann-Whitney rank sum test was used. Enumeration data were analyzed by the Chi-square test. Statistical analysis were performed using Python (version 3.7.4).

We used principal component analysis to reduce data dimensionality. In the case of retaining the information of the original data to the greatest extent, new features (principal component, PC) were reconstructed on the basis of the original features to reduce data redundancy and prevent the multicollinearity of the original features from affecting the reliability of logistic regression analysis. PCA calculation involves five steps: 1) The standardization was performed on each feature (Eq.1).

2) The covariance matrix (Eq.5) was calculated based on primary data (Eq.2). 3) Calculating the eigenvalues and eigenvector (Eq.6). Eq.3 means an original feature. Eq.4 means calculating the covariance of two features. 4) Calculating a set of eigenvectors and eigenvalues: $\left\{\left(\lambda_{1}, v_{1}\right),\left(\lambda_{2}, v_{2}\right) \cdots\left(\lambda_{i}, v_{i}\right)\right\}$, we sorted the eigenvalues in descending order and selected the top 25. 5) Projecting the primary data matrix to the new feature space (Eq.7). Factor loading method is presented as Eq.8.

$$
\begin{gathered}
x^{\prime}=\frac{x-\bar{x}}{s t d} \\
X=\left[\begin{array}{cccc}
x_{11} & x_{12} & \cdots & x_{1 k} \\
x_{21} & x_{22} & \cdots & x_{2 k} \\
\vdots & \vdots & \cdots & \vdots \\
x_{n 1} & x_{n 2} & \cdots & x_{n k}
\end{array}\right] \\
X_{k}=\begin{array}{rlll}
\left(x_{1 k}, x_{2 k}\right. & \cdots & \left.x_{n k}\right)^{T}
\end{array} \\
\operatorname{Cov}\left(X_{k-1}, X_{k}\right)=\frac{1}{n-1} \sum_{i=1}^{n}\left(X_{k-1}-\bar{X}_{k-1}\right)\left(X_{k}-\bar{X}_{k}\right)(4) \\
C=\left[\begin{array}{cccc}
\operatorname{Cov}\left(X_{1}, X_{1}\right) & \operatorname{Cov}\left(X_{1}, X_{2}\right) & \cdots & \operatorname{Cov}\left(X_{1}, X_{k}\right) \\
\operatorname{Cov}\left(X_{2}, X_{1}\right) & \operatorname{Cov}\left(X_{2}, X_{2}\right) & \cdots & \operatorname{Cov}\left(X_{2}, X_{k}\right) \\
\vdots & \vdots & \cdots & \vdots \\
\operatorname{Cov}\left(X_{k}, X_{1}\right) & \operatorname{Cov}\left(X_{k}, X_{2}\right) & \cdots & \operatorname{Cov}\left(X_{k}, X_{k}\right)
\end{array}\right](5)
\end{gathered}
$$




$$
\begin{gathered}
C v=\lambda v \\
X_{p c a}=\left[\begin{array}{c}
v_{1}^{T} \cdot X^{T} \\
v_{2}^{T} \cdot X^{T} \\
\vdots \\
v_{i}^{T} \cdot X^{T}
\end{array}\right]
\end{gathered}
$$

$L=v \sqrt{\lambda}$

\section{Results}

Table.1, Table.2, Table. 3 and Table. 4 show the basic information, laboratory examinations and tongue features among subjects with diabetes, prediabetes and normal. Using PCA to reduce the dimensions of the original data, the cumulative explained variance reached $91.9 \%$ and $91.8 \%$, respectively, and retained the information carried in the original data to the greatest extent (Figure.2 and Figure.8).

For the prediabetes group, PC_0, PC_4, PC_8, PC_15 and PC_20 are risk factors, PC_1 and PC_5 are protection factors (Table.5). The original features were associated with the PCs through factor loading method(Table.6). Figure. 3 showed the original features highly related to PC_0. Figure.4 shows the original features highly related to PC_1. Figure.5 shows the original features highly related to PC_4. Combining factor loadings can determine that TB-ASM, TC-ASM and TB-Cr are protective factors. TB-CON, TC-CON, TBMEAN, TC-MEAN, TB-ENT and TC-ENT are risk factors (Figure.6 and Figure.7).

Table.1 Gender composition

\begin{tabular}{llllll}
\hline Group & Female & Male & Total & $\chi^{2}$ & $P$ value \\
\hline Normal & 85 & 182 & 267 & 5.749 & 0.056 \\
\hline Prediabetes & 96 & 191 & 287 & & \\
Diabetes & 38 & 127 & 165 & & \\
Total & 219 & 500 & 719 & & \\
\hline
\end{tabular}

Table. 2 Basic information

\begin{tabular}{llll}
\hline Item & Normal & Prediabetes & Diabetes \\
\hline Age (years) & $49.985 \pm 9.456$ & $50.474 \pm 10.596$ & $52.182 \pm 10.557$ \\
\hline Height $(\mathrm{cm})$ & $168.157 \pm 8.069$ & $168.707 \pm 8.498$ & $169.139 \pm 7.798$ \\
\hline Weight $(\mathrm{kg})$ & $70.0(61.0,78.0)$ & $72.0(63.0,79.0)^{\star \star}$ & $75.0(66.0,82.0)^{\star \star \star}$ \\
BMl $\left(\mathrm{kg} / \mathrm{m}^{\wedge} 2\right)$ & $24.539(21.933,26.534)$ & $24.802(22.85,27.143)^{\star \star}$ & $25.952(23.939,27.757)^{\star \star \star}$ \\
SBP $(\mathrm{mmHg})$ & $128.202 \pm 17.383$ & $130.711 \pm 16.847^{\star \star \star}$ & $141.085 \pm 19.272^{\star \star \star}$ \\
DBP $(\mathrm{mmHg})$ & $80.0(73.0,88.0)$ & $81.0(75.0,88.0)^{\star \star \star}$ & $87.0(80.0,94.0)^{\star \star \star}$ \\
\hline
\end{tabular}

Note: Compared with normal group: ${ }^{\star \star} P<0.01,{ }^{\star \star \star} P<0.001$.

Table.3 Laboratory test results

\begin{tabular}{llll}
\hline Item & Normal & Prediabetes & Diabetes \\
\hline WBC $\left(10^{\wedge} 9 / \mathrm{L}\right)$ & $5.7(5.0,6.7)$ & $6.0(5.2,7.1)^{\star \star \star}$ & $6.6(5.7,7.9)^{\star \star \star \lambda}$ \\
\hline NE\# $\left(10^{\wedge} 9 / \mathrm{L}\right)$ & $3.3(2.7,4.05)$ & $3.5(2.8,4.1)^{\star \star \star}$ & $3.8(3.1,4.6)^{\star \star \star}$ \\
LY\# $\left(10^{\wedge} 9 / \mathrm{L}\right)$ & $1.8(1.5,2.25)$ & $2.0(1.6,2.3)^{\star}$ & $2.2(1.7,2.5)^{\star \star \star \star}$ \\
MO\# & $0.4(0.3,0.5)$ & $0.4(0.3,0.5)$ & $0.4(0.3,0.5)^{\star}$ \\
RBC $\left(10^{\wedge} 12 / L\right)$ & $4.82(4.515,5.145)$ & $4.83(4.52,5.12)^{\star \star}$ & $4.98(4.67,5.26)^{\star \star}$ \\
HCT $(\%)$ & $0.441(0.412,0.467)$ & $0.438(0.409,0.464)^{\star \star}$ & $0.45(0.423,0.468)^{\star}$ \\
\hline
\end{tabular}




\begin{tabular}{|c|c|c|c|}
\hline MCV (fL) & $90.9(88.6,93.45)$ & $90.8(88.4,92.9)$ & $90.5(87.7,93.5)$ \\
\hline RDW-CV (\%) & $13.1(12.7,13.5)$ & $13.1(12.8,13.6)$ & $13.0(12.6,13.4)$ \\
\hline HGB (g/L) & $149.0(138.0,159.0)$ & $149.0(138.0,157.0)^{\star \star}$ & $152.0(144.0,159.0)^{\star}$ \\
\hline $\mathrm{MCH}(\mathrm{pg})$ & $30.7(29.9,31.65)$ & $30.8(29.85,31.5)$ & $30.8(29.5,31.6)$ \\
\hline $\mathrm{MCHC}\left(10^{\wedge} 9 / \mathrm{L}\right)$ & $338.0(335.0,341.0)$ & $338.0(335.0,341.0)$ & $338.0(335.0,342.0)$ \\
\hline PLT (10^9/L) & $216.0(186.5,249.0)$ & $220.0(189.5,260.0)$ & $221.0(186.0,250.0)$ \\
\hline PCT (\%) & $0.19(0.17,0.21)$ & $0.19(0.17,0.23)$ & $0.19(0.17,0.22)$ \\
\hline MPV (fL) & $8.8(8.1,9.5)$ & $8.8(8.2,9.6)$ & $8.5(8.2,9.4)$ \\
\hline PDW (\%) & $16.5(16.2,16.9)$ & $16.6(16.3,17.0)$ & $16.6(16.3,17.1)$ \\
\hline $\mathrm{TCHO}(\mathrm{mmol} / \mathrm{L})$ & $5.31(4.77,5.98)$ & $5.56(4.8,6.105)$ & $5.38(4.69,6.07)$ \\
\hline $\mathrm{TG}(\mathrm{mmol} / \mathrm{L})$ & $1.36(0.97,2.06)$ & $1.55(1.085,2.235)^{\star \star \star}$ & $2.01(1.35,2.72)^{\star \star \star \star \Delta}$ \\
\hline LDL (mmol/L) & $3.143 \pm 0.796$ & $3.29 \pm 0.783$ & $3.116 \pm 0.907$ \\
\hline $\mathrm{HDL}(\mathrm{mmol} / \mathrm{L})$ & $1.31(1.12,1.56)$ & $1.28(1.1,1.5)^{\star \star \star}$ & $1.15(1.01,1.33)^{\star \star \star}$ \\
\hline UA (umol/L) & $368.0(305.0,442.0)$ & $379.0(323.5,440.0)$ & $376.0(309.0,439.0)$ \\
\hline $\operatorname{ALT}(\mathrm{U} / \mathrm{L})$ & $21.0(15.0,30.0)$ & $22.0(16.5,35.0)^{\star}$ & $26 \cdot 0(17.0,41.0)^{\star \star \star \star 力}$ \\
\hline GGT (U/L) & $25.0(18.0,40.0)$ & $30.0(19.0,43.0)^{\star \star \star}$ & $35.0(24.0,56.0)^{\star \star \star \star 厶}$ \\
\hline AST (U/L) & $23.0(19.0,27.0)$ & $23.0(19.0,28.0)$ & $23.0(19.0,32.0)$ \\
\hline ALP (U/L) & $78.0(67.0,92.5)$ & $76.0(62.5,89.0)$ & $79.0(68.0,95.0)$ \\
\hline TBA (umol/L) & $2.65(1.8,3.99)$ & $2.8(1.8,4.12)^{\star}$ & $3.2(2.2,4.8)^{\star \star}$ \\
\hline TBIL (umol/L) & $14.4(11.95,18.75)$ & $13.5(10.7,16.6)$ & 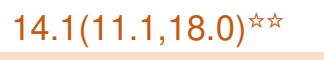 \\
\hline DBIL (umol/L) & $2.6(2.1,3.3)$ & $2.4(1.9,3.1)$ & $2 \cdot 6(2 \cdot 0,3 \cdot 2)^{2 \sqrt[1]{4}}$ \\
\hline IBIL (umol/L) & $11.7(9.65,15.45)$ & $10.9(8.85,13.6)$ & $11.6(9 \cdot 2,14.9)^{\text {质的 }}$ \\
\hline CREA (umol/L) & $74.0(63.0,85.0)$ & $76.0(64.0,85.0)$ & $74.0(60.0,83.0)$ \\
\hline BUN (mmol/L) & $5.01(4.295,5.995)$ & $5.23(4.405,6.14)$ & $5.29(4.6,6.3)^{\star}$ \\
\hline eGFR (ml/min*1.73m^2) & $97.7(88.45,108.45)$ & $96.45(86.4,107.3)^{\star \star}$ & $101.51(90.2,116.03)$ \\
\hline TP $(g / L)$ & $75.7(73.45,78.05)$ & $76.1(73.7,78.95)$ & $76.4(74.0,79.7)$ \\
\hline GLB (g/L) & $30.3(27.9,32.25)$ & $31.0(28.5,32.95)$ & 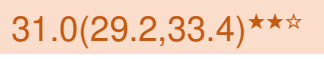 \\
\hline ALB (g/L) & $45.4(43.8,47.2)$ & $45.3(43.85,46.8)$ & $45.4(43.9,47.0)$ \\
\hline
\end{tabular}

Note: Compared with normal group: ${ }^{\star} P<0.05,{ }^{\star \star} P<0.01,{ }^{\star \star \star} P<0.001$; Compared with prediabetes group: ${ }^{\star} P<0.05$, ${ }^{\text {मे }} P<0.01$.

Table.4 Tongue features

\begin{tabular}{llll}
\hline Item & Normal & Prediabetes & Diabetes \\
\hline Per-All & $0.334(0.277,0.398)$ & $0.355(0.285,0.413)$ & $0.378(0.295,0.437)^{\star \star}$ \\
\hline Per-Part & $1.172(1.084,1.315)$ & $1.16(1.091,1.325)$ & $1.133(1.071,1.276)$ \\
\hline TB-CON & $68.675(55.111,86.709)$ & $72.534(56.031,90.586)^{\star}$ & $65.487(49.502,83.002)$ \\
\hline TB-ENT & $1.2(1.152,1.256)$ & $1.215(1.155,1.266)^{\star}$ & $1.19(1.127,1.247)$ \\
\hline TB-ASM & $0.079(0.07,0.09)$ & $0.077(0.068,0.089)^{\star}$ & $0.082(0.071,0.095)$ \\
\hline TB-MEAN & $0.025(0.023,0.028)$ & $0.026(0.023,0.029)^{\star}$ & $0.024(0.021,0.028)$ \\
\hline TC-CON & $76.007(54.91,102.513)$ & $84.678(60.534,113.175)^{\star}$ & $74.908(54.693,98.32)^{\star}$ \\
\hline TC-ENT & $1.227(1.153,1.298)$ & $1.253(1.177,1.314)^{\star}$ & $1.222(1.155,1.291)^{\star}$ \\
\hline TC-ASM & $0.073(0.061,0.087)$ & $0.069(0.059,0.082)^{\star}$ & $0.074(0.062,0.087)^{\star}$ \\
\hline TC-MEAN & $0.027(0.023,0.031)$ & $0.028(0.024,0.032)^{\star}$ & $0.027(0.023,0.031)^{\star}$ \\
\hline TB-R & $160.0(156.0,164.0)$ & $159.0(155.0,163.0)$ & $159.0(156.0,163.0)$ \\
\hline TB-G & $95.513 \pm 6.985$ & $96.143 \pm 7.723$ & $96.733 \pm 7.315$ \\
\hline TB-B & $99.0(94.0,105.0)$ & $100.0(95.0,105.0)$ & $100.0(96.0,107.0)$ \\
\hline TC-R & $151.0(142.0,158.0)$ & $152.0(144.0,158.0)$ & $151.0(145.0,157.0)$ \\
\hline TC-G & $105.0(96.0,113.0)$ & $107.0(98.0,114.0)$ & $106.0(99.0,113.0)$ \\
\hline TC-B & $107.0(97.0,116.0)$ & $109.0(101.0,117.0)$ & $109.0(101.0,115.0)$ \\
\hline TB-H & $356.274(354.699,358.551)$ & $356.114(354.525,357.843)$ & $355.767(354.084,358.26)$ \\
\hline & & & \\
\hline
\end{tabular}




\begin{tabular}{llll}
\hline TB-I & $118.0(114.0,122.0)$ & $118.0(114.0,122.0)$ & $119.0(115.0,122.0)$ \\
\hline TB-S & $0.194 \pm 0.022$ & $0.191 \pm 0.023$ & $0.187 \pm 0.024 \star \star$ \\
\hline TC-H & $358.25 \pm 4.285$ & $357.494 \pm 4.033$ & $357.291 \pm 4.412$ \\
\hline TC-I & $121.0(111.0,128.0)$ & $122.0(114.0,129.0)$ & $121.0(115.0,128.0)$ \\
\hline TC-S & $0.134(0.12,0.146)$ & $0.13(0.118,0.145)$ & $0.129(0.114,0.145)$ \\
\hline TB-L & $103.771(102.245,105.191)$ & $103.725(102.107,105.355)$ & $104.187(102.376,105.433)$ \\
\hline TB-a & $23.028 \pm 2.375$ & $22.702 \pm 2.503$ & $22.198 \pm 2.529 \star \star$ \\
\hline TB-b & $5.735(4.756,7.415)$ & $5.516(4.538,6.705)$ & $5.293(4.065,6.618)^{\star \star}$ \\
\hline TC-L & $105.679(102.422,108.271)$ & $106.219(103.312,108.49)$ & $106.019(103.322,108.121)$ \\
\hline TC-a & $15.518 \pm 2.148$ & $15.583 \pm 2.241$ & $15.302 \pm 2.286$ \\
\hline TC-b & $4.591 \pm 1.965$ & $4.21 \pm 1.748$ & $4.095 \pm 1.999 \star \star$ \\
\hline TB-Y & $115.204(111.43,118.37)$ & $114.839(111.325,118.412)$ & $115.815(111.441,118.745)$ \\
\hline TB-Cr & $156.11(154.583,157.367)$ & $155.61(153.996,157.177) \star$ & $155.171(152.964,156.845)^{\star \star \star \hbar}$ \\
\hline TB-Cb & $120.457(119.09,121.303)$ & $120.699(119.675,121.448)$ & $120.874(119.842,122.022)^{\star \star \star}$ \\
\hline TC-Y & $118.133(110.166,124.507)$ & $119.408(112.381,124.985)$ & $118.814(112.779,124.355)$ \\
\hline TC-Cr & $147.468(145.967,148.832)$ & $147.479(146.12,148.797)$ & $147.254(145.14,148.857)$ \\
\hline TC-Cb & $122.087 \pm 1.599$ & $122.337 \pm 1.475$ & $122.478 \pm 1.711$ \\
\hline
\end{tabular}

Note: Compared with normal group: ${ }^{\star} P<0.05,{ }^{\star \star} P<0.01,{ }^{\star \star \star} P<0.001$; Compared with prediabetes group: ${ }^{\star} P<0.05$.

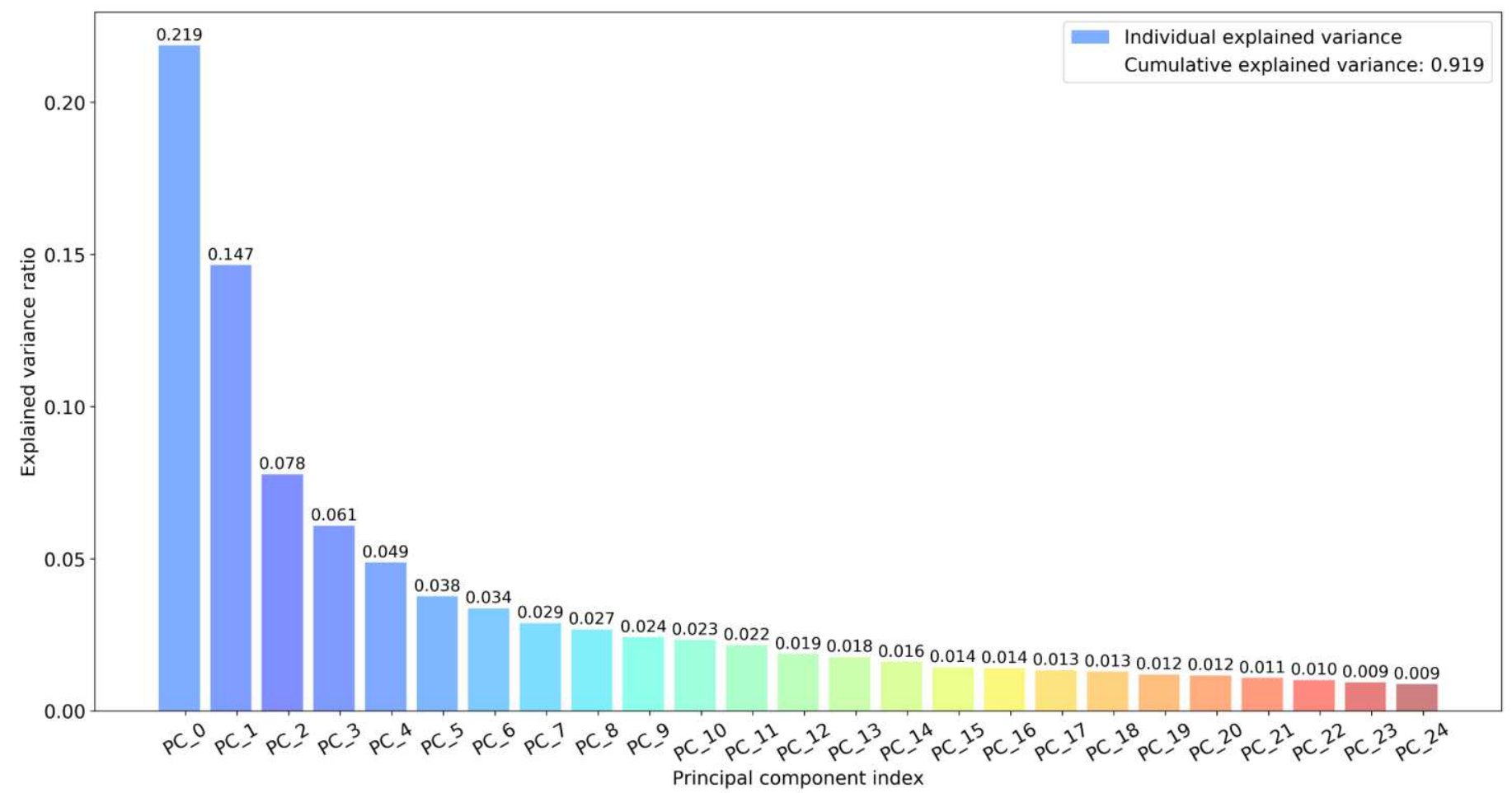

Figure.2 Proportion of variance explained by PCs in prediabetes group

Table.5 Logistic regression analysis for PCs in prediabetes group

\begin{tabular}{lcrrlcrc}
\hline Item & \multicolumn{1}{c}{ Coef } & \multicolumn{1}{c}{ Std err } & \multicolumn{1}{c}{$\boldsymbol{Z}$} & \multicolumn{1}{c}{ OR } & $\boldsymbol{P}$ value & Lower limit & Upper limit \\
\hline PC_0 & 0.058 & 0.023 & 2.513 & 1.059 & 0.012 & 0.013 & 0.102 \\
\hline PC_1 & -0.073 & 0.028 & -2.589 & 0.93 & 0.010 & -0.128 & -0.018 \\
\hline PC_2 & 0.055 & 0.038 & 1.438 & 1.057 & 0.150 & -0.020 & 0.131 \\
\hline PC_3 & 0.066 & 0.043 & 1.533 & 1.068 & 0.125 & -0.018 & 0.150 \\
\hline PC_4 & 0.171 & 0.050 & 3.441 & 1.187 & 0.001 & 0.074 & 0.269 \\
\hline PC_5 & -0.202 & 0.058 & -3.511 & 0.817 & 0.000 & -0.315 & -0.089 \\
PC_6 & -0.014 & 0.060 & -0.227 & 0.987 & 0.821 & -0.130 & 0.103 \\
\hline PC_7 & -0.065 & 0.062 & -1.049 & 0.937 & 0.294 & -0.186 & 0.056 \\
\hline
\end{tabular}




\begin{tabular}{lccccccc}
\hline PC_8 & 0.174 & 0.067 & 2.597 & 1.189 & 0.009 & 0.043 & 0.304 \\
\hline PC_9 & 0.056 & 0.069 & 0.815 & 1.058 & 0.415 & -0.079 & 0.191 \\
\hline PC_10 & 0.012 & 0.070 & 0.173 & 1.012 & 0.863 & -0.124 & 0.148 \\
\hline PC_11 & 0.013 & 0.073 & 0.182 & 1.013 & 0.855 & -0.129 & 0.156 \\
\hline PC_12 & 0.118 & 0.078 & 1.521 & 1.125 & 0.128 & -0.034 & 0.270 \\
\hline PC_13 & 0.040 & 0.080 & 0.499 & 1.041 & 0.618 & -0.117 & 0.197 \\
\hline PC_14 & -0.065 & 0.082 & -0.782 & 0.938 & 0.434 & -0.226 & 0.097 \\
\hline PC_15 & 0.199 & 0.089 & 2.228 & 1.22 & 0.026 & 0.024 & 0.373 \\
\hline PC_16 & -0.027 & 0.088 & -0.308 & 0.973 & 0.758 & -0.200 & 0.146 \\
\hline PC_17 & -0.129 & 0.092 & -1.400 & 0.879 & 0.161 & -0.310 & 0.052 \\
\hline PC_18 & 0.080 & 0.093 & 0.857 & 1.083 & 0.391 & -0.103 & 0.263 \\
\hline PC_19 & -0.043 & 0.098 & -0.435 & 0.958 & 0.664 & -0.234 & 0.149 \\
\hline PC_20 & 0.323 & 0.102 & 3.175 & 1.381 & 0.001 & 0.123 & 0.522 \\
\hline PC_21 & -0.004 & 0.105 & -0.035 & 0.996 & 0.972 & -0.209 & 0.202 \\
\hline PC_22 & 0.059 & 0.105 & 0.558 & 1.06 & 0.577 & -0.147 & 0.264 \\
\hline PC_23 & 0.007 & 0.108 & 0.062 & 1.007 & 0.950 & -0.205 & 0.218 \\
\hline PC_24 & -0.214 & 0.114 & -1.887 & 0.807 & 0.059 & -0.437 & 0.008 \\
\hline Const & 0.082 & 0.091 & 0.899 & 1.085 & 0.369 & -0.096 & 0.260 \\
\hline
\end{tabular}

Table.6 Factor loadings for PCs in prediabetes group

\begin{tabular}{llllllll}
\hline Item & PC_0 & PC_1 & PC_4 & PC_5 & PC_8 & PC_15 & PC_20 \\
\hline Gender & 0.476 & 0.121 & -0.025 & 0.155 & 0.096 & 0.129 & 0.025 \\
Age & -0.334 & 0.009 & -0.172 & -0.467 & -0.046 & 0.099 & -0.179 \\
BMI & 0.127 & 0.038 & 0.063 & -0.31 & -0.042 & -0.023 & -0.142 \\
SBP & -0.183 & 0.098 & 0.111 & -0.471 & -0.028 & 0.003 & -0.009 \\
DBP & -0.022 & 0.112 & 0.188 & -0.337 & -0.003 & -0.006 & 0.074 \\
WBC & 0.133 & 0.179 & 0.737 & 0.194 & -0.039 & -0.087 & 0.037 \\
NE\# & 0.052 & 0.186 & 0.626 & 0.226 & -0.113 & -0.147 & 0.093 \\
\hline LY\# & 0.195 & 0.021 & 0.491 & 0.004 & 0.103 & 0.076 & -0.188 \\
MO\# & 0.14 & 0.127 & 0.554 & 0.098 & -0.025 & 0.046 & -0.054 \\
EO\# & 0.163 & 0.14 & 0.194 & -0.022 & 0.21 & 0.057 & 0.312 \\
\hline BA\# & 0.07 & 0.06 & 0.359 & 0.077 & 0.145 & -0.112 & 0.154 \\
\hline RBC & 0.056 & 0.297 & 0.297 & 0.292 & 0.228 & 0.12 & -0.116 \\
\hline HCT & 0.111 & 0.314 & 0.222 & 0.124 & -0.024 & 0.072 & -0.119 \\
\hline MCV & -0.517 & 0.258 & -0.078 & -0.213 & -0.342 & -0.012 & -0.004 \\
RDW-CV & -0.595 & 0.207 & 0.066 & 0.033 & 0.177 & 0.15 & 0.026 \\
\hline HGB & 0.146 & 0.295 & 0.18 & 0.08 & -0.069 & 0.059 & -0.086 \\
MCH & -0.428 & 0.235 & -0.113 & -0.248 & -0.375 & -0.024 & 0.029 \\
\hline MCHC & -0.832 & 0.335 & -0.047 & -0.106 & -0.146 & 0.053 & 0.058 \\
\hline PLT & 0.076 & 0.072 & 0.449 & 0.326 & -0.111 & 0.254 & -0.081 \\
\hline PCT & 0.004 & 0.108 & 0.48 & 0.339 & -0.036 & 0.187 & -0.237 \\
MPV & -0.413 & 0.136 & -0.055 & -0.093 & 0.106 & -0.14 & -0.226 \\
\hline PDW & -0.641 & 0.246 & -0.061 & -0.161 & -0.022 & 0.006 & 0.109 \\
\hline TCHO & -0.094 & 0.07 & 0.245 & -0.247 & 0.513 & -0.13 & 0.015 \\
\hline TG & 0.317 & -0.02 & 0.184 & -0.33 & -0.047 & -0.113 & 0.211 \\
LDL & 0.039 & 0.033 & 0.241 & -0.186 & 0.542 & -0.103 & -0.124 \\
HDL & -0.55 & 0.102 & -0.058 & 0.196 & 0.101 & 0.068 & 0.099 \\
UA & 0.885 & 0.009 & -0.253 & -0.036 & 0.104 & -0.167 & 0.013 \\
\hline ALT & 0.327 & -0.005 & 0.147 & -0.17 & 0.016 & 0.216 & -0.075 \\
\hline & & & & & & &
\end{tabular}




\begin{tabular}{|c|c|c|c|c|c|c|c|}
\hline GGT & 0.382 & 0.101 & 0.202 & -0.201 & -0.075 & 0.012 & 0.312 \\
\hline AST & 0.171 & -0.004 & 0.083 & -0.235 & -0.021 & 0.231 & -0.089 \\
\hline ALP & -0.069 & 0.072 & 0.214 & -0.252 & -0.121 & -0.31 & -0.31 \\
\hline TBA & -0.013 & 0.057 & -0.001 & -0.128 & 0.029 & 0.31 & 0.064 \\
\hline TBIL & 0.095 & 0.041 & -0.38 & 0.506 & 0.04 & -0.059 & -0.005 \\
\hline DBIL & 0.014 & 0.037 & -0.442 & 0.551 & -0.055 & -0.001 & -0.022 \\
\hline IBIL & 0.109 & 0.041 & -0.36 & 0.487 & 0.058 & -0.07 & -0.002 \\
\hline CREA & 0.292 & 0.155 & -0.185 & 0.011 & 0.457 & 0.159 & -0.024 \\
\hline BUN & -0.115 & 0.101 & -0.146 & -0.252 & 0.391 & 0.216 & 0.184 \\
\hline eGFR & -0.367 & 0.087 & 0.261 & 0.264 & -0.458 & -0.112 & 0.12 \\
\hline TP & -0.586 & 0.256 & 0.247 & -0.004 & 0.071 & -0.218 & 0.145 \\
\hline GLB & -0.372 & 0.119 & 0.204 & -0.17 & 0.1 & -0.214 & 0.043 \\
\hline ALB & -0.499 & 0.275 & 0.147 & 0.217 & -0.011 & -0.087 & 0.187 \\
\hline Per-All & 0.001 & -0.463 & 0.068 & -0.086 & -0.229 & 0.105 & 0.091 \\
\hline Per-Part & -0.231 & 0.355 & -0.075 & 0.191 & 0.054 & -0.155 & 0.074 \\
\hline TB-CON & -0.032 & -0.538 & 0.089 & -0.017 & -0.123 & -0.105 & -0.067 \\
\hline TB-ENT & -0.462 & -0.355 & 0.086 & -0.003 & -0.11 & -0.067 & -0.04 \\
\hline TB-ASM & -0.272 & 0.601 & -0.061 & -0.016 & 0.061 & 0.133 & 0.029 \\
\hline TB-MEAN & -0.144 & -0.508 & 0.085 & -0.01 & -0.122 & -0.107 & -0.05 \\
\hline TC-CON & -0.048 & -0.664 & 0.094 & 0.028 & -0.022 & 0.107 & 0.053 \\
\hline TC-ENT & -0.435 & -0.558 & 0.114 & 0.033 & -0.011 & 0.131 & 0.083 \\
\hline TC-ASM & -0.183 & 0.736 & -0.096 & -0.059 & -0.043 & -0.057 & -0.091 \\
\hline TC-MEAN & -0.15 & -0.661 & 0.101 & 0.04 & -0.005 & 0.111 & 0.078 \\
\hline TB-R & -0.876 & -0.032 & -0.072 & 0.077 & 0.174 & -0.1 & -0.106 \\
\hline TB-G & -0.664 & -0.471 & -0.116 & 0.026 & 0.082 & -0.069 & -0.031 \\
\hline TB-B & -0.56 & -0.552 & -0.022 & 0.039 & 0.138 & -0.131 & -0.036 \\
\hline TC-R & -0.583 & -0.624 & 0.084 & 0.099 & 0.125 & 0.017 & 0.035 \\
\hline TC-G & -0.46 & -0.8 & 0.081 & 0.027 & 0.029 & 0.066 & -0.004 \\
\hline TC-B & -0.393 & -0.821 & 0.113 & 0.046 & 0.083 & 0.016 & 0.009 \\
\hline TB-H & -0.908 & 0.348 & -0.014 & -0.014 & -0.053 & 0.096 & 0.007 \\
\hline TB-I & -0.773 & -0.365 & -0.079 & 0.05 & 0.144 & -0.108 & -0.064 \\
\hline TB-S & -0.196 & 0.693 & 0.107 & 0.032 & 0.032 & 0.042 & -0.074 \\
\hline TC-H & -0.885 & 0.36 & -0.009 & -0.027 & -0.08 & 0.112 & -0.003 \\
\hline TC-I & -0.491 & -0.767 & 0.093 & 0.06 & 0.082 & 0.031 & 0.014 \\
\hline TC-S & -0.189 & 0.793 & -0.077 & 0.083 & 0.084 & -0.069 & 0.055 \\
\hline TB-L & -0.96 & -0.023 & -0.046 & 0.016 & 0.042 & -0.0 & -0.026 \\
\hline TB-a & -0.222 & 0.625 & 0.123 & 0.051 & 0.091 & -0.02 & -0.066 \\
\hline TB-b & -0.411 & 0.455 & -0.163 & -0.003 & -0.065 & 0.113 & -0.032 \\
\hline TC-L & -0.841 & -0.44 & 0.086 & 0.031 & 0.021 & 0.082 & 0.013 \\
\hline TC-a & -0.186 & 0.643 & -0.003 & 0.133 & 0.208 & -0.14 & 0.086 \\
\hline TC-b & -0.268 & 0.485 & -0.132 & -0.015 & -0.112 & 0.116 & -0.015 \\
\hline TB-Y & -0.824 & -0.304 & -0.09 & 0.043 & 0.115 & -0.078 & -0.056 \\
\hline TB-Cr & -0.851 & 0.458 & 0.026 & 0.018 & 0.02 & 0.045 & -0.035 \\
\hline TB-Cb & -0.877 & 0.244 & 0.083 & -0.021 & -0.036 & 0.064 & 0.027 \\
\hline TC-Y & -0.555 & -0.732 & 0.088 & 0.051 & 0.063 & 0.051 & 0.01 \\
\hline $\mathrm{TC}-\mathrm{Cr}$ & -0.881 & 0.397 & 0.02 & 0.046 & 0.04 & 0.036 & 0.037 \\
\hline TC-Cb & -0.88 & 0.264 & 0.058 & -0.02 & -0.019 & 0.054 & 0.008 \\
\hline
\end{tabular}




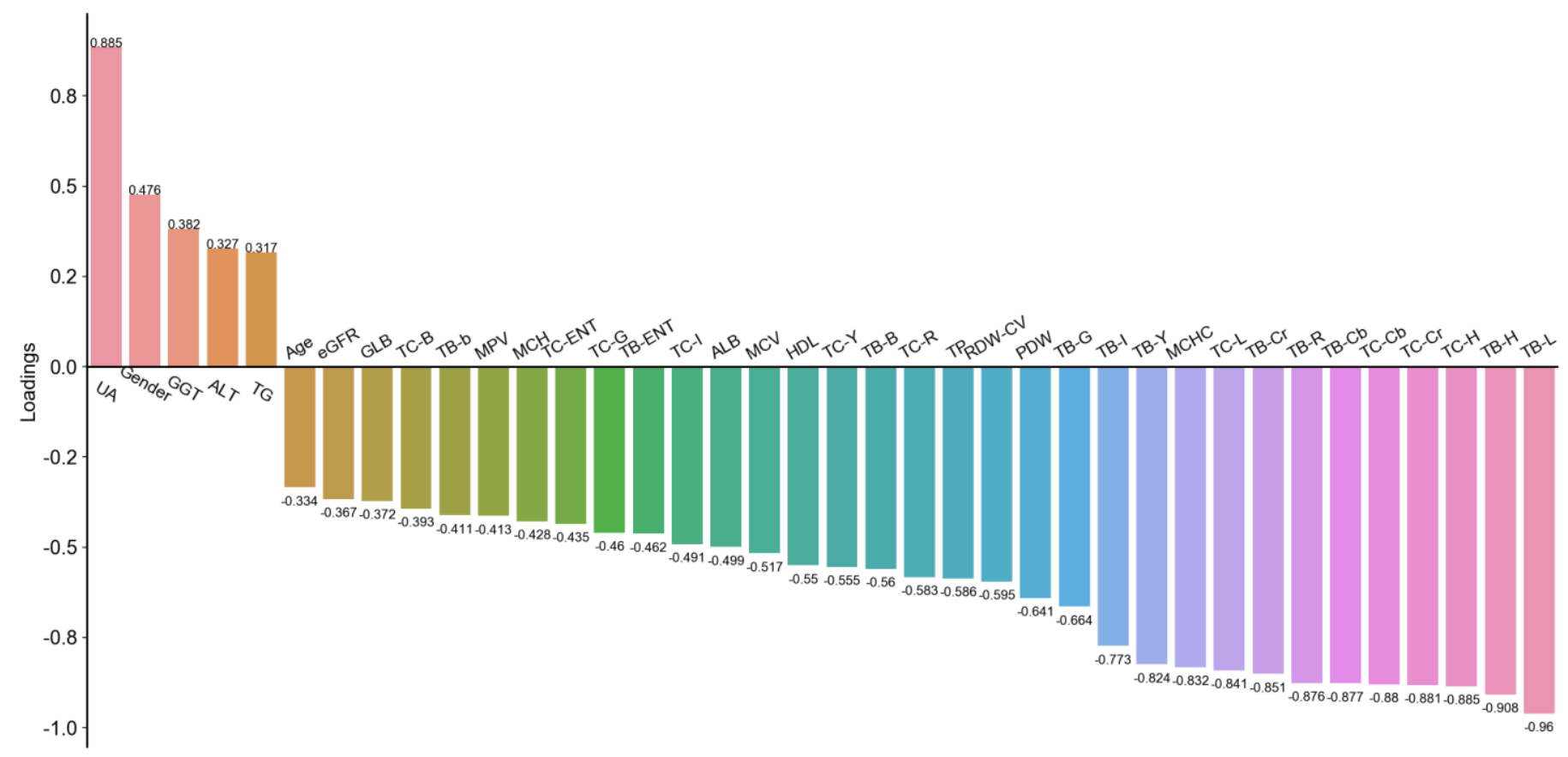

Figure.3 Factor loadings of PC_0 in prediabetes group

Note: TB-Cr, TB-ENT, TC-ENT are negatively correlated with the risk factor PC_0, and the correlations are $-0.851,-0.462$, and -0.435 , respectively.

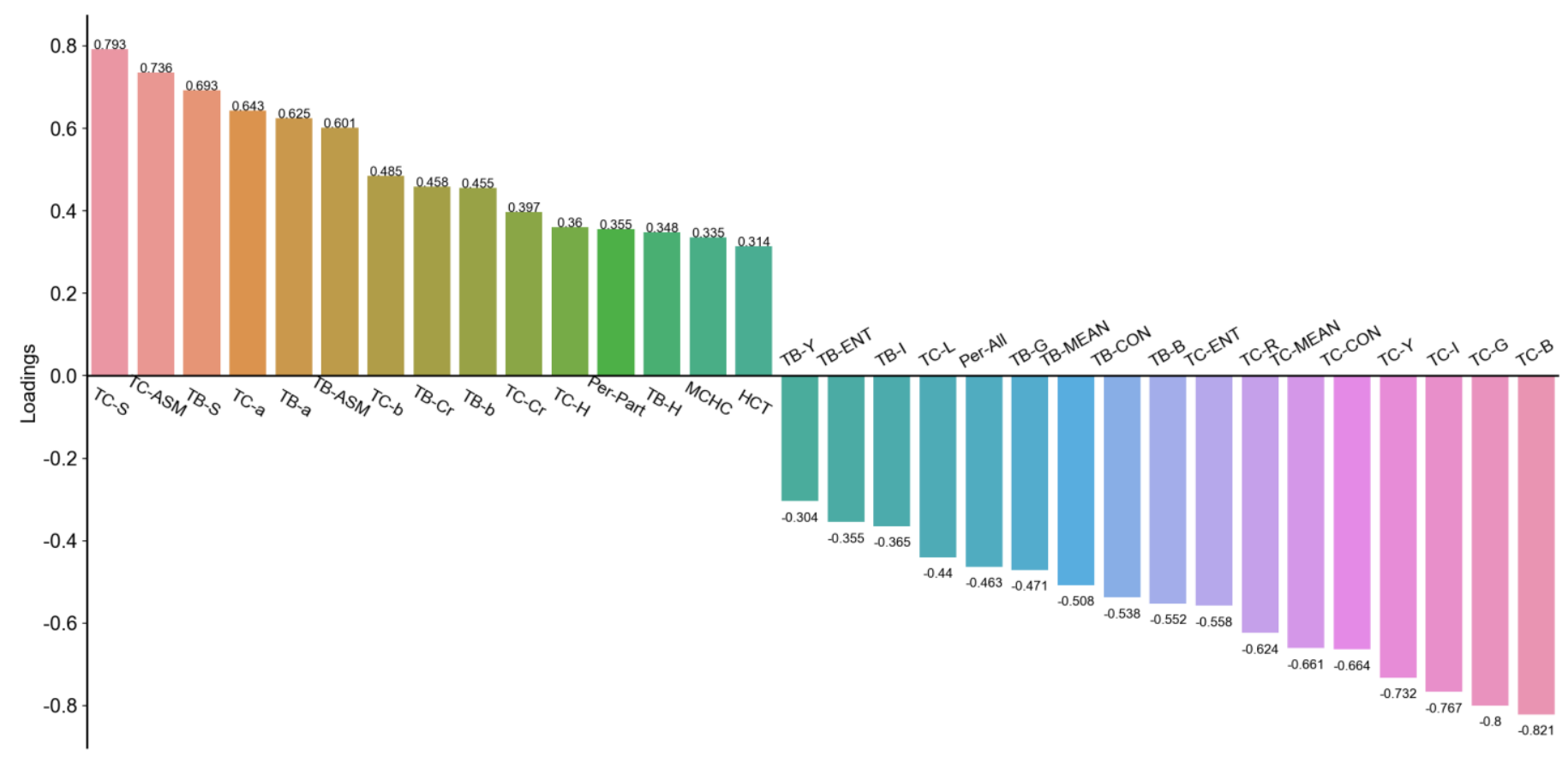

Figure.4 Factor loadings of PC_1 in prediabetes group

Note: PC 1 is a protective factor, TC-ASM, TB-ASM, TB-Cr and PC 1 are positively correlated, and the correlation coefficients are $0.736,0.601$ and 0.458 , respectively. TCCON, TC-MEAN, TC-ENT, TB-CON, TB-MEAN, TB-ENT are negatively correlated with PC_1, the correlation coefficients are -0.664, $-0.661,-0.558,-0.538,-0.508,-0.355$, respectively. 


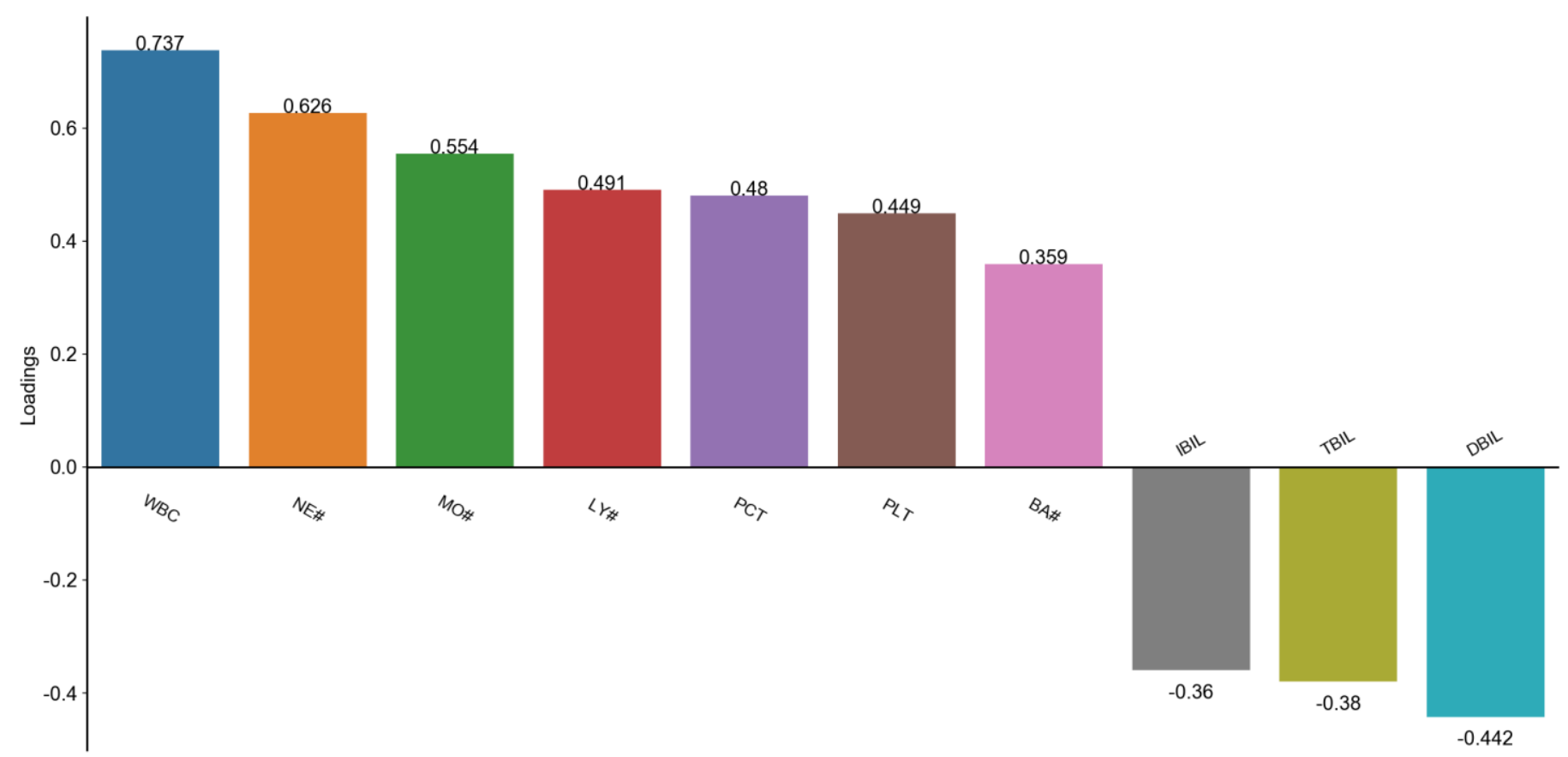

Figure.5 Factor loadings of PC_4 in prediabetes group

Note: PC_4 is a risk factor, WBC, NE\#, LY\# are positively correlated with PC_4, and the correlation coefficients are $0.737,0.626,0.491$, respectively.

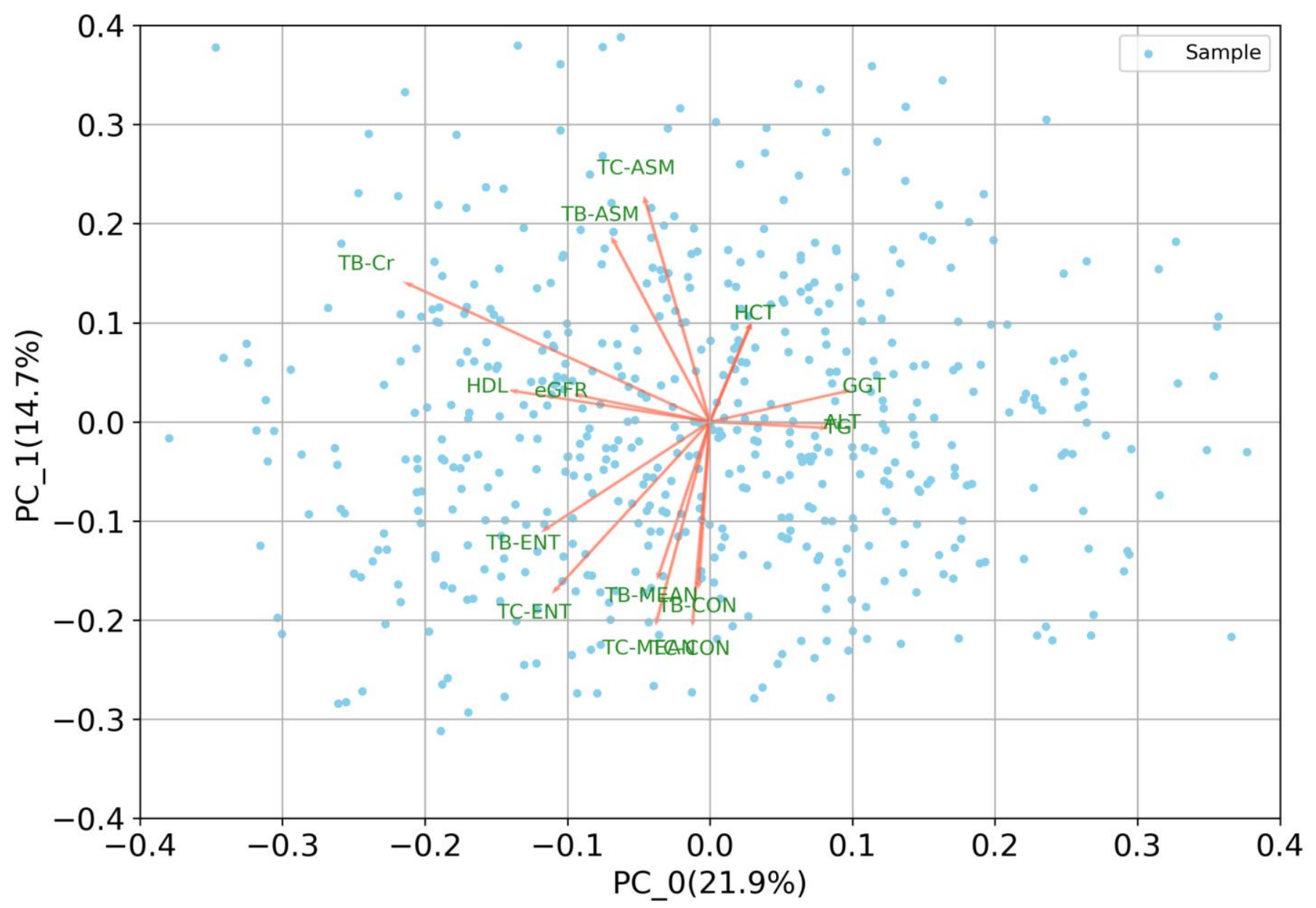

Figure.6 Biplot of PC_0 and PC_1 in prediabetes group

Note: A biplot shows how strongly each feature influences a principal component. The position coordinates of the feature are determined by the weight of feature of the corresponding PC. The angle between features indicates the correlation between features. An acute angle $\left(<90^{\circ}\right)$ indicates a positive correlation between two features. Right angles $\left(90^{\circ}\right)$ indicate irrelevance. Obtuse angles $\left(>90^{\circ}\right)$ indicate negative correlation. PC_0 is a risk factor, TB-Cr mainly acts on PC_0 (The vector that is sub-parallel to the $\mathrm{X}$-axis), and TB-Cr has a reverse effect on PC_0 and is a protective factor. PC_1 is a protective factor, TB-ASM, TC-ASM, TB-CON, TC-CON, TB-MEAN, TC-MEAN, TCENT mainly act on PC_1 (The vectors that are sub-parallel to the y-axis). TB-ASM and TC-ASM play a positive role on PC_1 and are protective factors; TB-CON, TC-CON, 


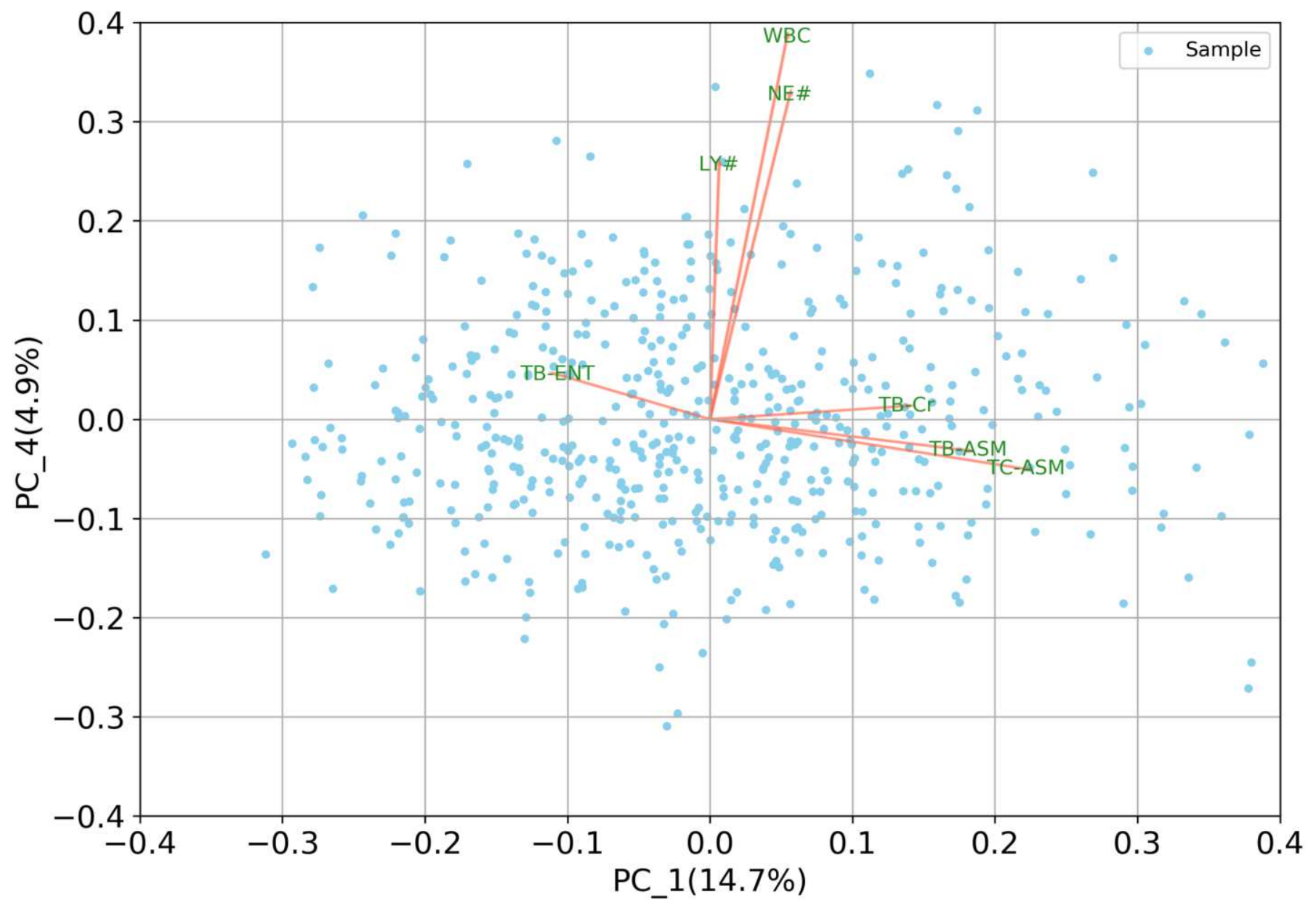

Figure.7 Biplot of PC_1 and PC_4 in prediabetes group

Note: PC_1 is a protective factor, TB-ENT mainly act on PC_1 (The vector that is sub-parallel to the $\mathrm{x}$-axis). TB-ENT plays a negative role on PC_1 and is risk factor.

For the diabetes group, PC_0, PC_3, PC_4, PC_5, PC_9, PC_11, PC_19 were risk factors. PC_2, PC_18, PC_21 and PC_24 are protective factors (Table.7). The original features were associated with the PCs through the factor loading method (Table.8). Figure.9 shows the original features highly related to PC_0, Figure.10 shows the original features highly related to PC_2, and Figure.11 shows the original features highly related to PC_3. Figure.12, Figure.13, and Figure.14 respectively calculated the position and orientation of key tongue features in the coordinate system composed of PC_0 and PC_2, PC_0 and PC_3, PC_2 and PC_3. Combined with factor loading analysis, it can be determined that TB-a, TB-S, TB-Cr, TB-b and TC-b are protective factors, and Per-all and TB-Cb are risk factors. 


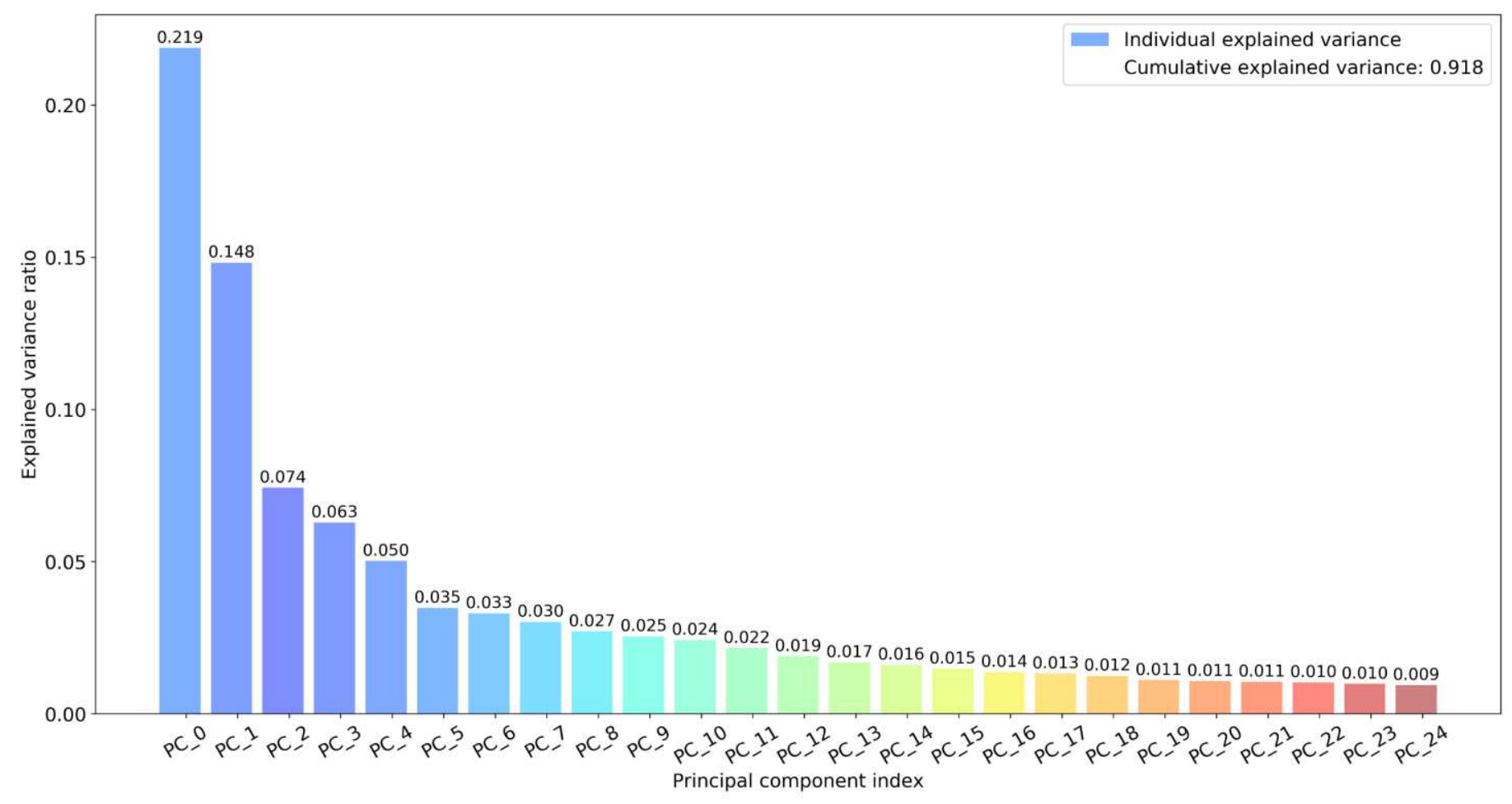

Figure.8 Proportion of variance explained by PCs in diabetes group

Table.7 Logistic regression analysis for PCs in diabetes group

\begin{tabular}{lrrrrrrr}
\hline Item & \multicolumn{1}{c}{ Coef } & Std err & \multicolumn{1}{c}{ Z } & OR & P value & Lower limit & Upper limit \\
\hline PC_0 & 0.139 & 0.035 & 3.982 & 1.149 & 0.000 & 0.071 & 0.208 \\
\hline PC_1 & -0.052 & 0.038 & -1.370 & 0.95 & 0.171 & -0.125 & 0.022 \\
\hline PC_2 & -0.188 & 0.057 & -3.298 & 0.829 & 0.001 & -0.299 & -0.076 \\
\hline PC_3 & 0.294 & 0.059 & 5.004 & 1.342 & 0.000 & 0.179 & 0.410 \\
\hline PC_4 & 0.214 & 0.062 & 3.451 & 1.238 & 0.001 & 0.092 & 0.335 \\
\hline PC_5 & 0.453 & 0.081 & 5.559 & 1.572 & 0.000 & 0.293 & 0.612 \\
\hline PC_6 & -0.027 & 0.087 & -0.305 & 0.974 & 0.760 & -0.198 & 0.145 \\
\hline PC_7 & 0.005 & 0.084 & 0.059 & 1.005 & 0.953 & -0.159 & 0.169 \\
\hline PC_8 & 0.083 & 0.088 & 0.940 & 1.086 & 0.347 & -0.090 & 0.256 \\
\hline PC_9 & 0.182 & 0.089 & 2.048 & 1.199 & 0.041 & 0.008 & 0.355 \\
\hline PC_10 & -0.062 & 0.092 & -0.665 & 0.94 & 0.506 & -0.242 & 0.120 \\
\hline PC_11 & 0.310 & 0.099 & 3.127 & 1.364 & 0.002 & 0.116 & 0.505 \\
\hline PC_12 & -0.130 & 0.105 & -1.244 & 0.878 & 0.214 & -0.336 & 0.075 \\
\hline PC_13 & -0.049 & 0.111 & -0.439 & 0.953 & 0.661 & -0.265 & 0.168 \\
\hline PC_14 & -0.196 & 0.116 & -1.688 & 0.822 & 0.091 & -0.424 & 0.032 \\
\hline PC_15 & 0.064 & 0.118 & 0.542 & 1.066 & 0.588 & -0.167 & 0.295 \\
\hline PC_16 & -0.146 & 0.118 & -1.232 & 0.864 & 0.218 & -0.378 & 0.086 \\
\hline PC_17 & 0.054 & 0.121 & 0.441 & 1.055 & 0.659 & -0.184 & 0.291 \\
\hline PC_18 & -0.368 & 0.128 & -2.873 & 0.692 & 0.004 & -0.618 & -0.117 \\
\hline PC_19 & 0.388 & 0.142 & 2.725 & 1.474 & 0.006 & 0.109 & 0.667 \\
\hline PC_20 & 0.227 & 0.135 & 1.680 & 1.254 & 0.093 & -0.038 & 0.491 \\
\hline PC_21 & -0.375 & 0.136 & -2.762 & 0.688 & 0.006 & -0.640 & -0.109 \\
\hline PC_22 & -0.069 & 0.137 & -0.506 & 0.933 & 0.613 & -0.337 & 0.199 \\
\hline PC_23 & 0.197 & 0.139 & 1.415 & 1.217 & 0.157 & -0.076 & 0.469 \\
\hline PC_24 & -0.417 & 0.147 & -2.840 & 0.659 & 0.005 & -0.705 & -0.129 \\
\hline Const & -0.687 & 0.126 & -5.463 & 0.503 & 0.000 & -0.933 & -0.440 \\
\hline & & & & & & & \\
\hline
\end{tabular}


Table 8 Factor loadings for original features in diabetes group

\begin{tabular}{|c|c|c|c|c|c|c|c|c|c|c|c|}
\hline Item & PC_0 & PC_2 & PC_3 & PC_4 & PC_5 & PC_9 & PC_11 & PC_18 & PC_19 & PC_21 & PC_24 \\
\hline Gender & 0.427 & 0.301 & 0.508 & -0.241 & -0.096 & 0.016 & -0.036 & -0.003 & 0.039 & 0.208 & 0.052 \\
\hline Age & -0.299 & 0.163 & -0.155 & -0.021 & 0.308 & 0.226 & -0.042 & -0.106 & 0.084 & 0.211 & -0.148 \\
\hline BMI & 0.073 & -0.01 & 0.325 & 0.105 & 0.295 & 0.161 & 0.224 & -0.169 & -0.033 & -0.239 & -0.129 \\
\hline SBP & -0.086 & 0.042 & 0.145 & 0.24 & 0.55 & 0.268 & 0.371 & 0.139 & -0.027 & 0.036 & -0.01 \\
\hline DBP & 0.059 & 0.034 & 0.241 & 0.222 & 0.485 & 0.202 & 0.423 & 0.179 & -0.013 & -0.035 & 0.198 \\
\hline WBC & 0.135 & -0.203 & 0.538 & 0.536 & -0.269 & 0.158 & 0.04 & -0.108 & -0.129 & -0.027 & 0.012 \\
\hline NE\# & 0.059 & -0.147 & 0.482 & 0.428 & -0.3 & 0.098 & 0.002 & -0.023 & -0.074 & 0.003 & -0.004 \\
\hline LY\# & 0.18 & -0.18 & 0.267 & 0.425 & -0.031 & 0.13 & 0.123 & -0.296 & -0.284 & -0.066 & 0.029 \\
\hline MO\# & 0.159 & -0.187 & 0.408 & 0.351 & -0.178 & 0.227 & 0.057 & -0.091 & -0.083 & 0.081 & 0.016 \\
\hline EO\# & 0.125 & -0.088 & 0.219 & 0.158 & -0.001 & 0.194 & -0.096 & 0.24 & 0.491 & -0.148 & -0.01 \\
\hline BA\# & 0.034 & -0.136 & 0.359 & 0.247 & -0.082 & 0.126 & -0.005 & 0.44 & 0.023 & 0.059 & 0.088 \\
\hline $\mathrm{RBC}$ & -0.046 & 0.283 & 0.576 & 0.144 & -0.178 & -0.075 & 0.014 & 0.083 & 0.148 & -0.011 & -0.055 \\
\hline HCT & -0.0 & 0.445 & 0.679 & 0.099 & -0.053 & -0.194 & -0.14 & 0.087 & 0.018 & -0.012 & -0.053 \\
\hline MCV & -0.564 & 0.257 & 0.186 & -0.036 & 0.165 & -0.188 & -0.193 & -0.033 & -0.141 & -0.02 & 0.027 \\
\hline RDW-CV & -0.587 & -0.26 & -0.111 & 0.049 & -0.076 & 0.271 & 0.125 & -0.065 & 0.164 & 0.29 & 0.133 \\
\hline HGB & 0.025 & 0.475 & 0.68 & 0.063 & -0.01 & -0.227 & -0.135 & 0.072 & 0.021 & -0.034 & -0.063 \\
\hline $\mathrm{MCH}$ & -0.494 & 0.306 & 0.214 & -0.073 & 0.204 & -0.235 & -0.189 & -0.046 & -0.13 & -0.053 & 0.01 \\
\hline $\mathrm{MCHC}$ & -0.879 & 0.156 & 0.126 & -0.046 & 0.094 & -0.131 & -0.006 & -0.086 & 0.021 & -0.067 & 0.007 \\
\hline PLT & 0.03 & -0.466 & -0.108 & 0.44 & -0.469 & -0.036 & 0.186 & -0.024 & 0.079 & -0.042 & -0.003 \\
\hline РCT & -0.059 & -0.465 & -0.106 & 0.46 & -0.423 & -0.007 & 0.094 & 0.093 & -0.159 & -0.026 & -0.113 \\
\hline MPV & -0.432 & 0.099 & 0.042 & -0.062 & 0.193 & 0.053 & -0.179 & 0.154 & -0.347 & 0.034 & -0.153 \\
\hline PDW & -0.738 & 0.076 & 0.142 & -0.056 & 0.121 & -0.013 & 0.034 & -0.065 & 0.033 & 0.025 & 0.029 \\
\hline $\mathrm{TCHO}$ & -0.048 & -0.02 & -0.052 & 0.473 & 0.152 & -0.1 & -0.301 & -0.116 & 0.053 & 0.17 & 0.043 \\
\hline $\mathrm{TG}$ & 0.311 & -0.024 & 0.142 & 0.176 & 0.25 & -0.199 & 0.252 & -0.317 & 0.143 & 0.042 & 0.138 \\
\hline LDL & -0.006 & 0.009 & -0.001 & 0.388 & 0.049 & -0.025 & -0.439 & -0.05 & -0.013 & 0.141 & -0.091 \\
\hline HDL & -0.525 & -0.029 & -0.247 & 0.055 & -0.106 & 0.079 & -0.096 & 0.373 & -0.055 & 0.013 & 0.116 \\
\hline UA & 0.744 & 0.141 & 0.014 & -0.235 & -0.021 & 0.011 & -0.082 & 0.022 & -0.138 & 0.0 & 0.031 \\
\hline ALT & 0.489 & -0.099 & 0.044 & 0.116 & 0.35 & 0.116 & -0.118 & -0.015 & 0 & -0 . & 0.043 \\
\hline GGT & 0.467 & -0.103 & 0.089 & 0.119 & 0.283 & -0.077 & -0.126 & -0.11 & 0.247 & 0.004 & -0.052 \\
\hline AST & 0.305 & -0.084 & 0.035 & 0.165 & 0.426 & 0.169 & -0.168 & -0.022 & -0.042 & -0.065 & 0.117 \\
\hline ALP & 0.013 & -0.025 & 0.162 & 0.026 & 0.276 & 0.095 & 0.059 & 0.057 & -0.098 & 0.123 & -0.056 \\
\hline TBA & 0.03 & -0.114 & 0.095 & 0.01 & 0.351 & 0.092 & 0.19 & 0.158 & -0.151 & 0.323 & -0.24 \\
\hline TBIL & 0.053 & 0.564 & 0.216 & -0.237 & -0.322 & 0.308 & 0.142 & -0.083 & -0.002 & 0.006 & -0.051 \\
\hline DBIL & -0.022 & 0.519 & 0.184 & -0.335 & -0.336 & 0.338 & 0.118 & -0.008 & -0.01 & -0.008 & -0.055 \\
\hline IBIL & 0.067 & 0.561 & 0.218 & -0.213 & -0.313 & 0.296 & 0.144 & -0.096 & 0.0 & 0.009 & -0.049 \\
\hline CREA & 0.212 & 0.329 & 0.189 & -0.324 & -0.008 & 0.375 & -0.114 & -0.07 & -0.042 & 0.076 & 0.277 \\
\hline BUN & -0.048 & 0.2 & 0.017 & -0.146 & 0.165 & 0.26 & -0.08 & 0.025 & 0.049 & -0.425 & -0.297 \\
\hline eGFR & -0.362 & -0.243 & 0.15 & 0.241 & -0.084 & -0.429 & 0.163 & 0.081 & 0.021 & 0.044 & -0.11 \\
\hline TP & -0.572 & 0.005 & 0.035 & 0.362 & 0.072 & 0.031 & 0.019 & 0.05 & 0.073 & -0.085 & -0.043 \\
\hline GLB & -0.333 & -0.205 & -0.137 & 0.403 & 0.149 & 0.213 & -0.012 & -0.052 & 0.098 & -0.085 & -0.154 \\
\hline ALB & -0.532 & 0.259 & 0.224 & 0.101 & -0.064 & -0.21 & 0.046 & 0.146 & -0.0 & -0.036 & 0.119 \\
\hline Per-All & 0.067 & -0.068 & 0.505 & -0.085 & 0.066 & -0.22 & 0.074 & -0.102 & 0.085 & -0.025 & 0.127 \\
\hline Per-Part & -0.245 & 0.214 & -0.245 & 0.021 & -0.108 & 0.191 & -0.01 & -0.068 & 0.207 & 0.212 & -0.124 \\
\hline TB-CON & 0.083 & 0.545 & -0.264 & 0.208 & -0.021 & -0.141 & 0.191 & 0.0 & 0.015 & 0.1 & -0.158 \\
\hline TB-ENT & -0.458 & 0.598 & -0.198 & 0.253 & -0.015 & -0.123 & 0.183 & -0.033 & 0.028 & 0.008 & -0.067 \\
\hline TB-ASM & -0.31 & -0.537 & 0.206 & -0.206 & 0.028 & 0.101 & -0.165 & -0.024 & -0.015 & 0.027 & 0.097 \\
\hline
\end{tabular}




\begin{tabular}{|c|c|c|c|c|c|c|c|c|c|c|c|}
\hline TB-MEAN & -0.107 & 0.627 & -0.255 & 0.256 & -0.018 & -0.14 & 0.211 & -0.015 & 0.017 & 0.035 & -0.095 \\
\hline TC-CON & -0.006 & 0.488 & -0.26 & 0.322 & -0.007 & 0.008 & -0.001 & 0.037 & -0.055 & -0.02 & 0.146 \\
\hline TC-ENT & -0.429 & 0.464 & -0.169 & 0.327 & 0.013 & 0.034 & -0.041 & 0.011 & -0.029 & -0.059 & 0.154 \\
\hline TC-ASM & -0.227 & -0.398 & 0.177 & -0.292 & -0.006 & -0.081 & 0.07 & -0.066 & 0.03 & 0.066 & -0.109 \\
\hline TC-MEAN & -0.131 & 0.482 & -0.234 & 0.334 & 0.003 & 0.039 & -0.028 & 0.035 & -0.047 & -0.05 & 0.167 \\
\hline TB-R & -0.864 & -0.118 & -0.083 & -0.143 & -0.035 & 0.043 & 0.083 & 0.046 & -0.037 & -0.003 & -0.02 \\
\hline TB-G & -0.599 & -0.299 & 0.144 & -0.289 & -0.004 & -0.05 & 0.146 & 0.018 & -0.014 & -0.037 & 0.015 \\
\hline TB-B & -0.469 & -0.164 & 0.309 & -0.243 & 0.048 & -0.073 & 0.14 & 0.048 & -0.001 & 0.032 & -0.026 \\
\hline TC-R & -0.525 & -0.11 & 0.064 & 0.044 & -0.039 & 0.228 & -0.216 & -0.024 & -0.001 & -0.023 & -0.028 \\
\hline TC-G & -0.359 & -0.147 & 0.153 & 0.011 & -0.017 & 0.131 & -0.154 & -0.027 & 0.012 & 0.027 & -0.052 \\
\hline TC-B & -0.29 & -0.091 & 0.227 & 0.038 & 0.009 & 0.131 & -0.171 & -0.013 & 0.019 & 0.031 & -0.041 \\
\hline TB-H & -0.935 & -0.01 & -0.012 & 0.005 & -0.01 & -0.012 & 0.014 & -0.071 & 0.018 & -0.027 & 0.051 \\
\hline TB-I & -0.728 & -0.209 & 0.121 & -0.243 & 0004 & -0.024 & 0.133 & 0.04 & -0.019 & 0.0 & -0.013 \\
\hline TB-S & -0.322 & 0.291 & -0.166 & 0.241 & 0.008 & 0.071 & -0.073 & -0.018 & 0.007 & 0.072 & -0.024 \\
\hline TC-H & -0.915 & -0.001 & -0.023 & -0.004 & -0.011 & -0.027 & 0.036 & -0.07 & 0.018 & -0.008 & 0.028 \\
\hline TC-I & -0.403 & -0.119 & 0.151 & 0.031 & -0.017 & 0.167 & -0.187 & -0.021 & 0.009 & 0.011 & -0.041 \\
\hline TC-S & -0.33 & 0.101 & -0.202 & 0.022 & -0.012 & 0.062 & 0.05 & 0.017 & -0.01 & -0.096 & 0.095 \\
\hline TB-L & -0.952 & -0.119 & 0.091 & -0.133 & 0.001 & -0.031 & 0.085 & -0.023 & 0.003 & -0.016 & 0.025 \\
\hline TB-a & -0.345 & 0.308 & -0.171 & 0.225 & -0.005 & 0.09 & -0.086 & 0.022 & -0.006 & 0.072 & -0.049 \\
\hline TB-b & -0.437 & -0.14 & -0.435 & 0.009 & -0.111 & 0.095 & -0.023 & -0.046 & -0.034 & -0.109 & 0.059 \\
\hline TC-L & -0.795 & -0.083 & 0.139 & 0.035 & -0.011 & 0.11 & -0.136 & -0.055 & 0.021 & 0.009 & -0.017 \\
\hline TC-a & -0.316 & 0.165 & -0.119 & 0.072 & -0.012 & 0.133 & -0.064 & 0.014 & -0.015 & -0.083 & 0.073 \\
\hline TC-b & -0.31 & -0.102 & -0.351 & -0.067 & -0.092 & 0.031 & 0.075 & -0.034 & -0.033 & -0.059 & 0.004 \\
\hline TB-Y & -0.79 & -0.223 & 0.092 & -0.236 & -0.008 & -0.024 & 0.128 & 0.024 & -0.018 & -0.019 & 0.003 \\
\hline TB-Cr & -0.897 & 0.085 & -0.071 & 0.061 & -0.011 & 0.026 & -0.007 & -0.035 & 0.005 & 0.007 & 0.015 \\
\hline TB-Cb & -0.905 & 0.099 & 0.227 & 0.029 & 0.055 & -0.066 & 0.024 & -0.064 & 0.045 & 0.034 & 0.026 \\
\hline TC-Y & -0.472 & -0.128 & 0.14 & 0.026 & -0.02 & 0.161 & -0.176 & -0.029 & 0.011 & 0.011 & -0.041 \\
\hline TC-Cr & -0.923 & 0.048 & -0.027 & 0.04 & -0.012 & 0.061 & -0.04 & -0.053 & 0.01 & -0.041 & 0.047 \\
\hline TC-Cb & -0.904 & 0.081 & 0.175 & 0.04 & 0.047 & -0.057 & 0.016 & -0.057 & 0.04 & 0.02 & 0.043 \\
\hline
\end{tabular}

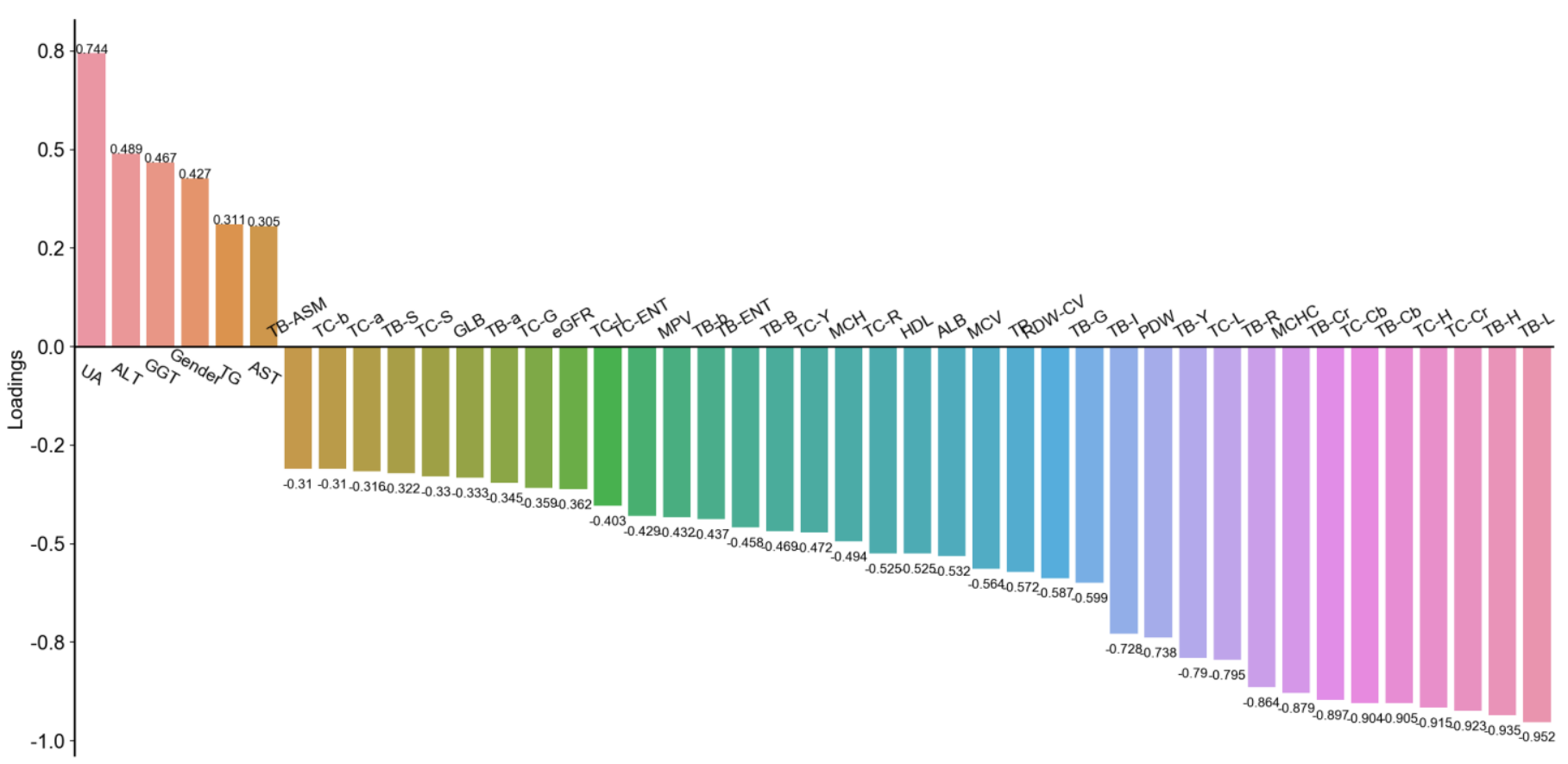

Figure.9 Factor loadings of PC_0 in diabetes group

Note: PC_0 is a risk factor, TB-Cb, TB-Cr, TB-b, TB-a, TB-S, TC-b are negatively correlated with PC_0, and the correlation coefficients are $-0.905,-0.897,-0.437,-0.345,-$

$0.322,-0.31$, respectively. 


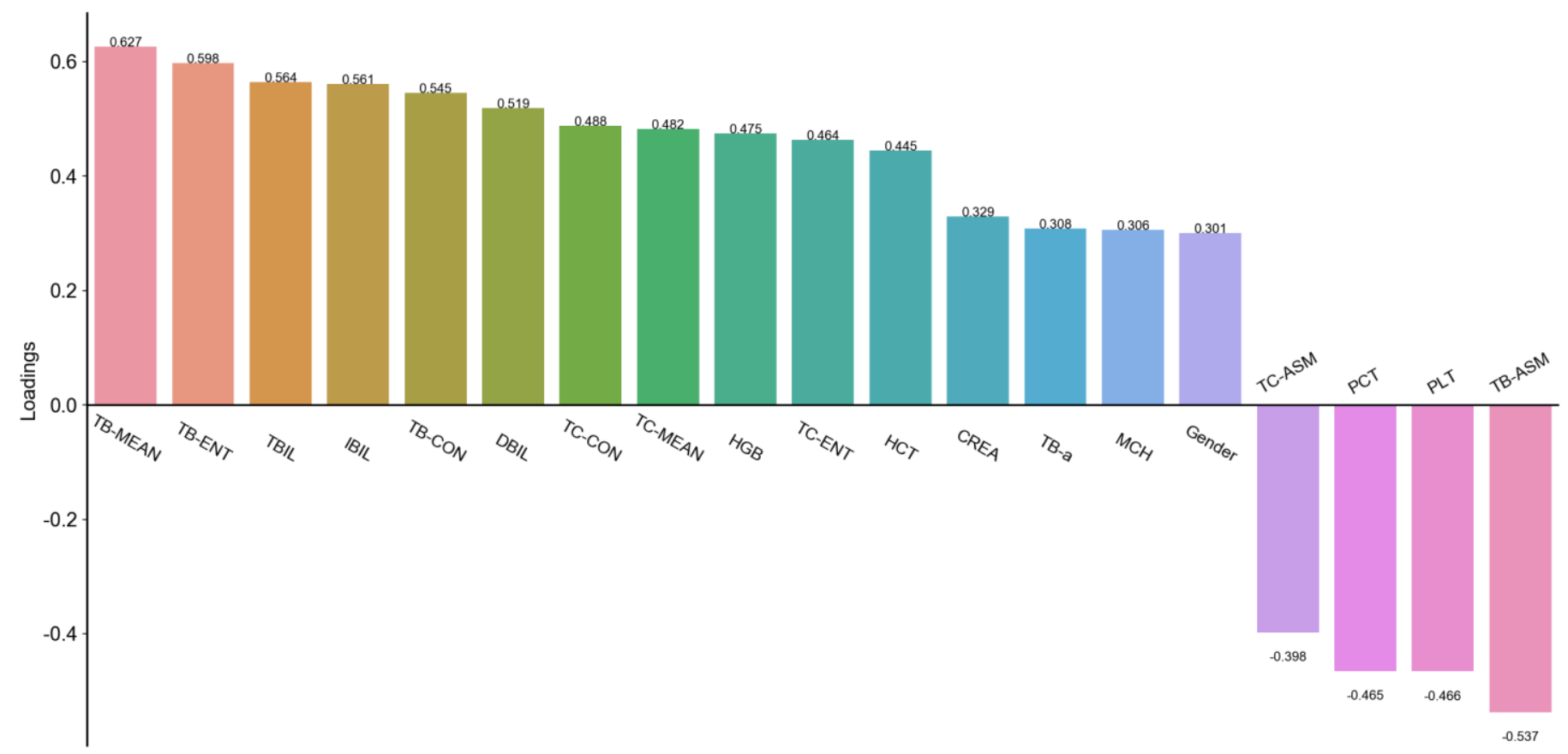

Figure.10 Factor loadings of PC_2 in diabetes group

Note: PC_2 is a protective factor, TB-a is positively correlated with PC_2, and the correlation coefficient is 0.308 .

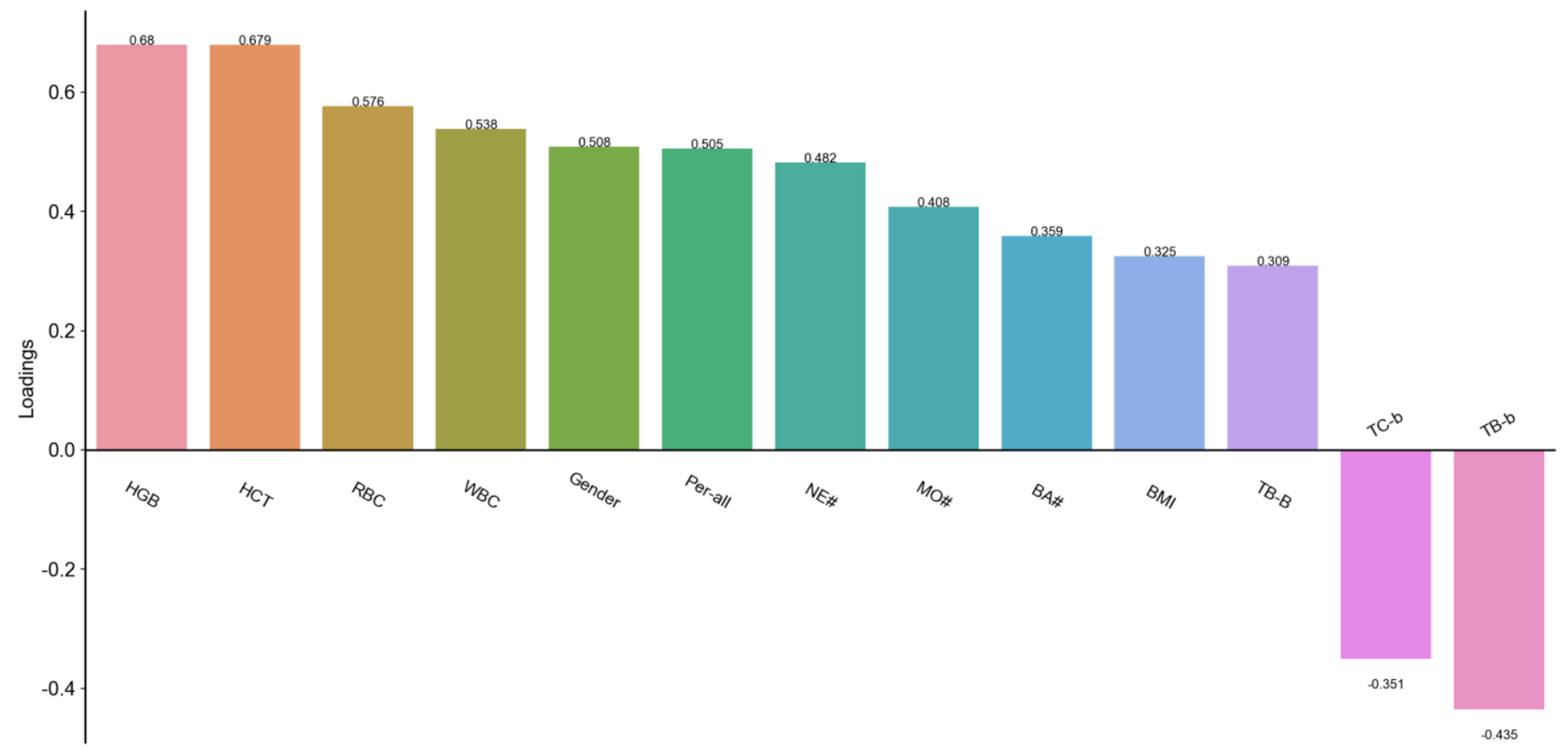

Figure.11 Factor loadings of PC_3 in diabetes group

Note: PC_3 is a risk factor, TB-b and TC-b are negatively correlated with PC_3, and the correlation coefficients are -0.435 and -0.351 , respectively. 


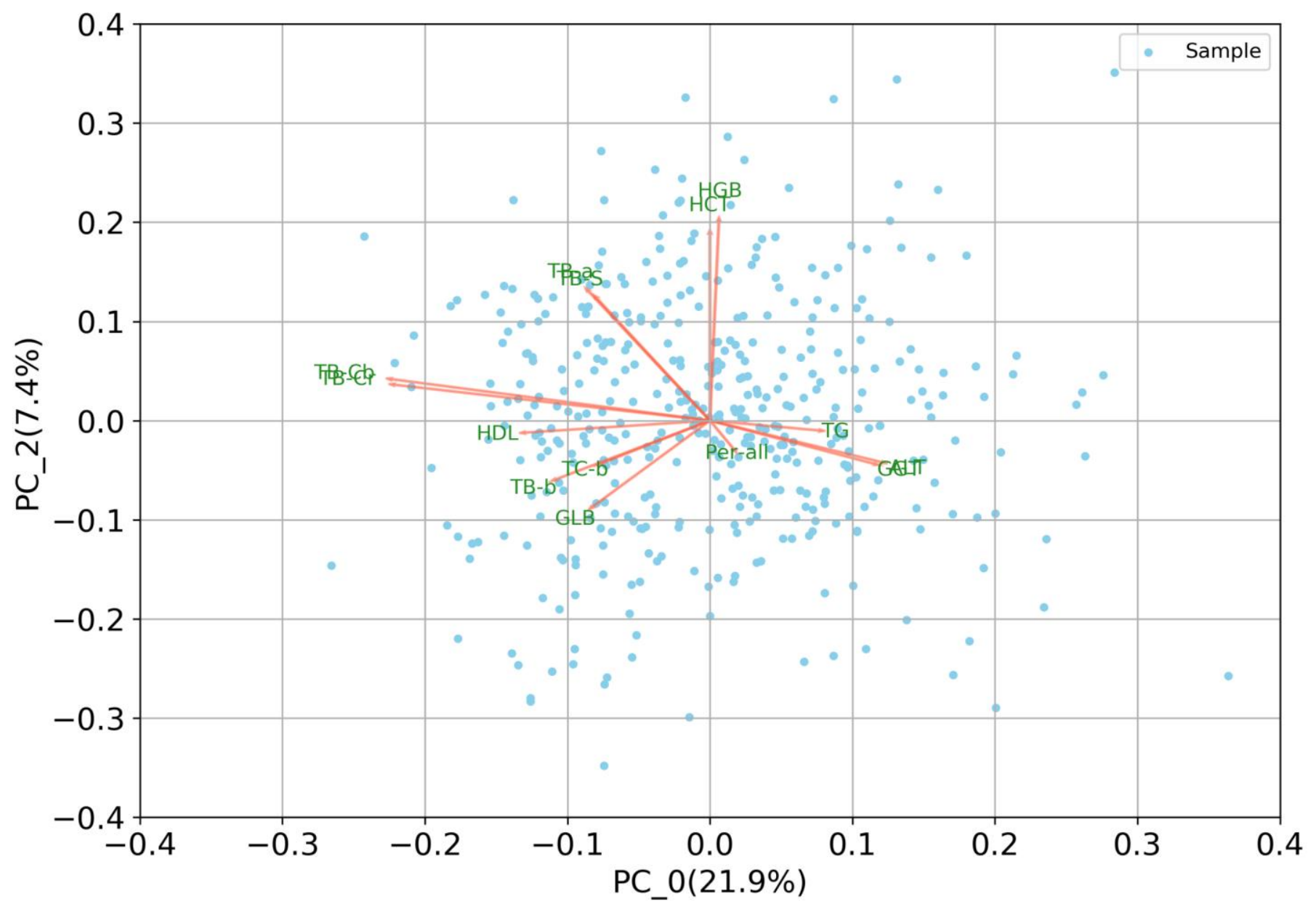

Figure.12 Biplot of PC_0 and PC_2 in diabetes group

Note: PC_0 is a risk factor, TB-Cr, TB-b, TC-b mainly act on PC_0 (The vectors that are sub-parallel to the $\mathrm{x}$-axis), TB-Cr, TB-b and TC-b play a negative role on PC_0 and are protective factors. PC_2 is a protective factor, TB-a and TB-S mainly act on PC_2 (The vectors that are sub-parallel to the y-axis), TB-a and TB-S positively act on PC_2 as a protective factor. 


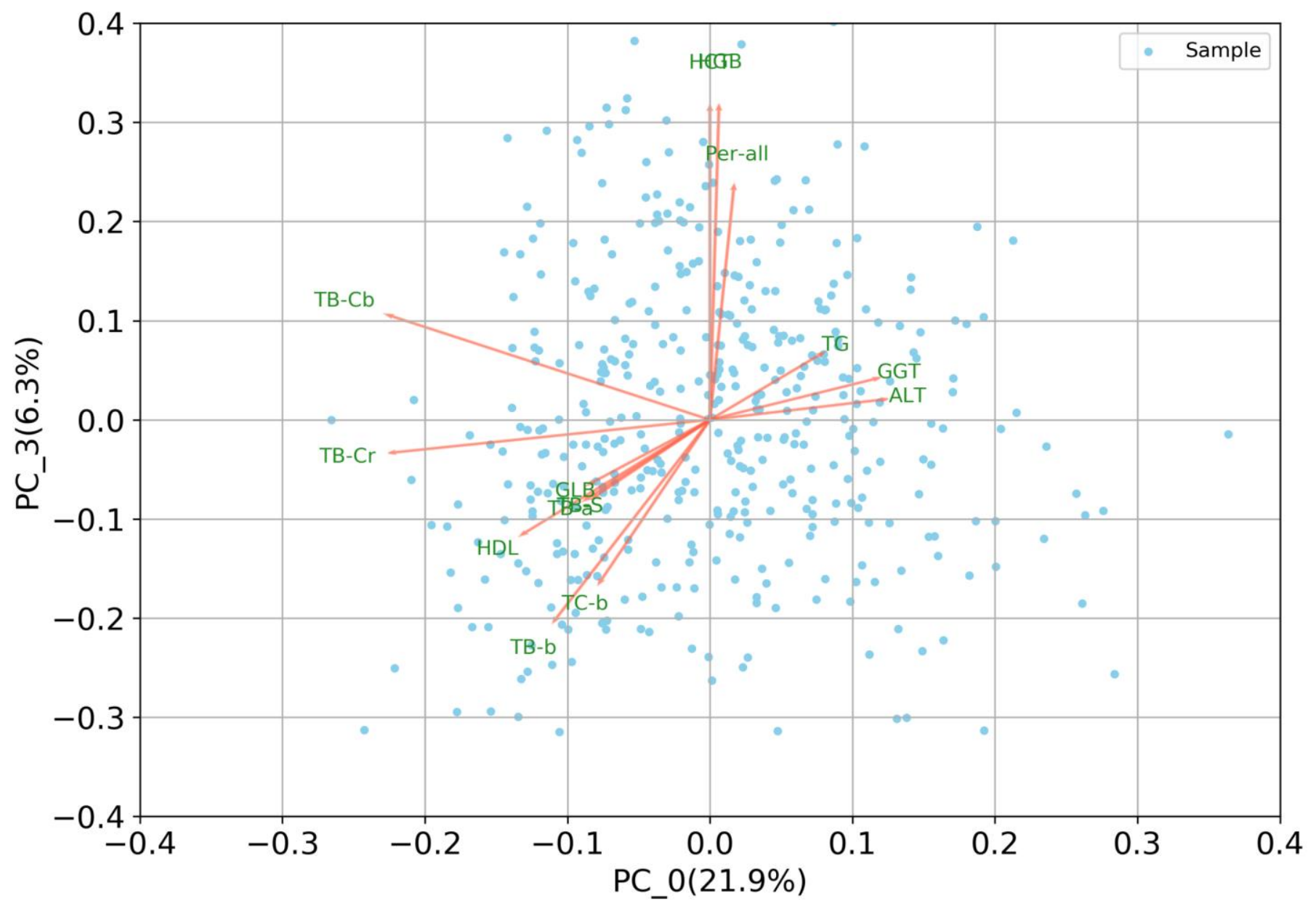

Figure.13 Biplot of PC_0 and PC_3 in diabetes group

Note: PC_3 is a risk factor, Per-all mainly acts on PC_3 (The vector that is sub-parallel to the y-axis), Per-all has a positive effect on PC_ 3 and is a risk factor. 


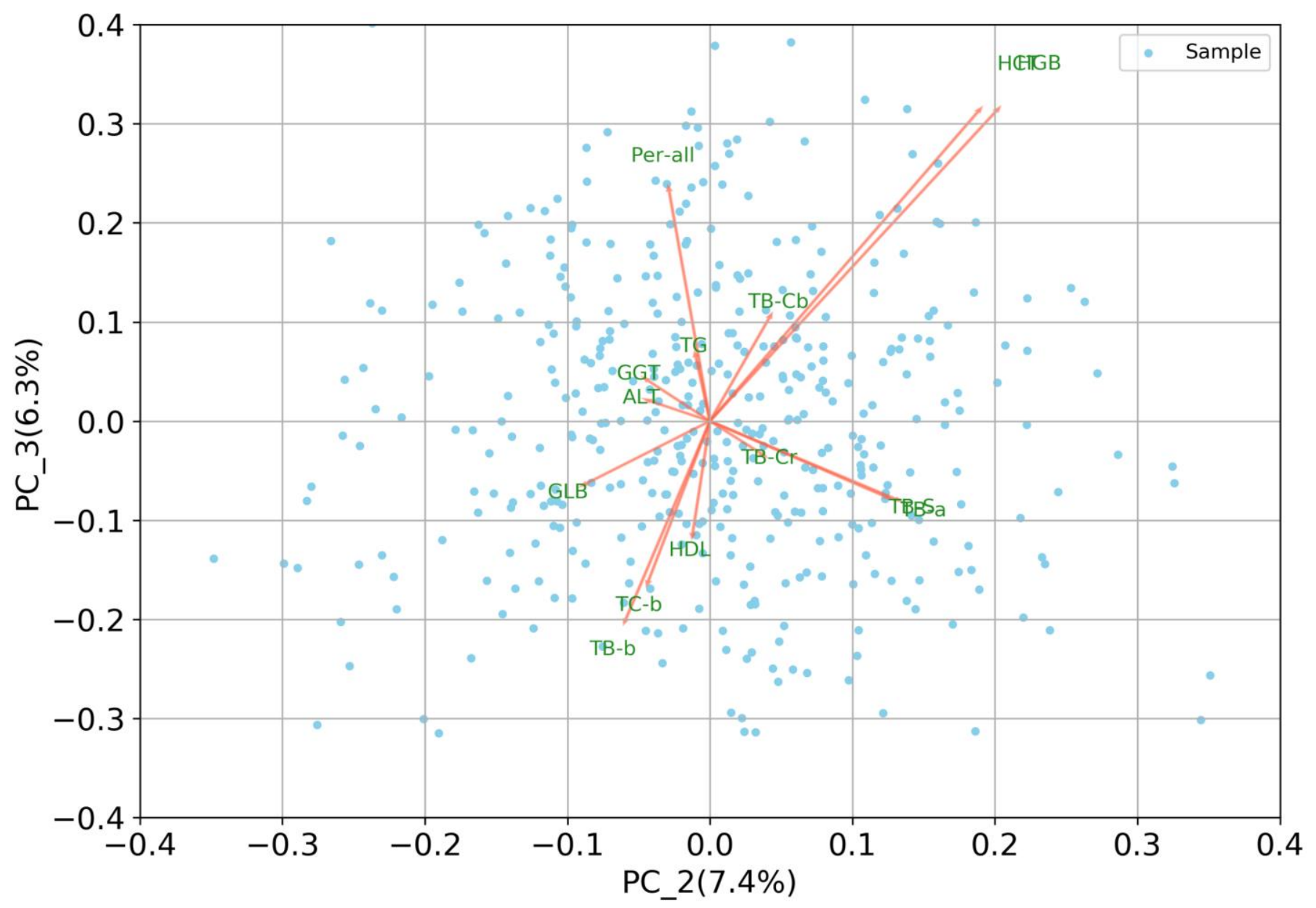

Figure.14 Biplot of PC_2 and PC_3 in diabetes group

Note: PC_3 is a risk factor, TB-Cb mainly acts on PC_3 (The vector that is sub-parallel to the y-axis), TB-Cb has a positive effect on PC_3 and is a risk factor.

\section{Discussion}

Through PCA combined with logistic regression, the key tongue features and changing laws during the onset of diabetes were analyzed. In the prediabetic group, TB-ASM, TC-ASM and TB-Cr are protective factors. As these indicators increase, the risk of prediabetes decreases. TB-CON, TC-CON, TB-MEAN, TC-MEAN, TB-ENT and TC-ENT are risk factors. As these indicators increase, the risk of prediabetes increases. At the same time, this also suggests that in the early stage of the disorder of glucose metabolism, the texture features are the first to change, and these texture features reflect the tenderness of the tongue and the moisturizing of the tongue coating. When prediabetes occurs, the texture of the tongue will become rough, and the tongue coating will be dry, which manifests as TB-CON, TC-CON, TB-MEAN, TC-MEAN, TB-ENT and TC-ENT increase, and TB-ASM And TC-ASM decreased. In the diabetes group, TB-Cb and Per-all are risk factors. As these indicators increase, the risk of diabetes increases. TB-Cr, TB-a, TB-S, TB-b and TC-b are protective factors. As these indicators increase, the risk of diabetes decreases. Entering the diabetes stage, the color features begin to change, manifested as the red component of the tongue decreases, the blue component increases, and the blue component of the tongue coating increases. In addition, the increase of Per-all indicates that the tongue coating increases and thickens. Tongue features include both protective factors and risk factors, and they have a bidirectional predictive effect on prediabetes and diabetes. In the prediabetic population, the texture feature was found to have an early warning effect; in the diabetic population, the color feature and the tongue coating 
ratio feature were found to have an early warning effect.

Individuals with prediabetes, especially those with Impaired Glucose Tolerance (IGT) or abnormal HbA1c (6.0\% - 6.4\%), not only are at increased risk of developing diabetes, but also have an increased risk of cardiovascular Diseases ${ }^{[12]}$. For individuals with prediabetes, those later diagnosed with diabetes had medical expenditures nearly $1 / 3$ higher than those who were not later diagnosed ${ }^{[13]}$. The best way to reverse the epidemic of diabetes is to intervene in prediabetes as early as possible [14]. For patients with undiagnosed potential diabetes, the insidious onset and lack of awareness of diabetes will lead to

missed diagnosis and poor prognosis ${ }^{[15,16]}$. Therefore, it is very important to develop reliable diabetes risk warning tools. Tongue features contain rich physiological and pathological information, which can be obtained non-invasively, and are closely related to glucose metabolism, showing potential advantages ${ }^{[17]}$. Therefore, finding the key tongue features related to diabetes risk and clarifying the clinical significance of the changes in these tongue features will provide a basis for the application of tongue features to diabetes risk prediction.

Logistic regression analysis requires that the sample size should be more than 15 times the size of independent variables, and there is no multicollinearity between the independent variables. By introducing PCA, we reduced the data dimension to 25 features (PCs) to eliminat the collinearity of the original features. PCA makes full use of original data and ensures the reliability of analysis conclusions. We used the PCA combined with logistic regression analysis method to find the key tongue features reflecting glucose metabolism, and clarified the significance of the changes in these tongue features. However, tongue features are continuous measurement data, and classification standards need to be established in the future to facilitate future clinical practice. This study did not consider the simultaneous increase of fasting blood glucose and HbA1c. This may mean that the patient's glucose metabolism is more disordered.

\section{Conclusion}

In this study, PCA was first used to reduce the dimensionality of the original data and retain more than $90 \%$ of the original data information. 25 new features were reconstructed on the basis of 76 original features. Then use logistic regression to calculate the OR value of the new feature. Next, the factor loading method is used to associate the original feature with the new feature. Through this study, the tongue features that are highly related to the risk of diabetes have been determined, and the clinical significance of these changes in tongue features have been clarified. This will lay the foundation for the study of constructing an early warning model of diabetes risk based on the tongue features.

\section{Declarations}

Ethics approval and consent to participate We confirm that all experiments were performed in accordance with relevant guidelines and regulations. All subjects signed an informed consent form. The study was approved by the IRB of Shuguang Hospital affiliated with Shanghai University of TCM.

Consent for publication Not applicable.

Availability of data and materials The datasets used and/or analysed during the current study available from the 
corresponding author on reasonable request.

Competing interests The authors declare that they have no competing interests.

Funding This study was supported by the National Key Research and Development Program of China [grant numbers 2017YFC1703300 and 2017YFC1703301]; National Natural Science Foundation of China [grant numbers 81873235, 81973750, and 81904094]; and 1226 major Project [grant number BWS17J028]. The study sponsors were not involved in the design of the study, the collection, analysis, and interpretation of data, writing the report or the decision to submit the report for publication.

Authors' contributions Jun Li wrote the whole manuscript text, analyzing the data, coding the program and plotting the results. These drafts were revised for important scientific content by Jiacai Li, Ji Cui, Changle Zhou and Jiatuo Xu. Xiaojuan $\mathrm{Hu}$ and Liping Tu helped in the ideas of study. Yulin Shi, Jiacai Li and Zijuan Bi provided assistance in data collection and analysis. All authors gave final approval of the version to be published. Jiatuo Xu is the guarantor of this work.

Acknowledgements We thank all investigators, study teams and subjects for participating in these studies. We thank the doctors at the Shuguang Hospital for their assistance in this study. The authors gratefully acknowledge the financial supports by the National Key Research and Development Program of China[grant numbers 2017YFC1703300 and 2017YFC1703301]; National Natural Science Foundation of China [grant numbers 81873235, 81973750, and 81904094]; and 1226 major Project [grant number BWS17J028].

\section{References}

[1] TABáK A G, HERDER C, RATHMANN W, et al. Prediabetes: a high-risk state for diabetes development [J]. Lancet, 2012, 379 (9833): 2279-90.

[2] BARDINI G, DICEMBRINI I, CRESCI B, et al. Inflammation markers and metabolic characteristics of subjects with 1-h plasma glucose levels [J]. Diabetes care, 2010, 33(2): 411-3.

[3] CAMPBELL M D, SATHISH T, ZIMMET P Z, et al. Benefit of lifestyle-based T2DM prevention is influenced by prediabetes phenotype [J]. Nature Reviews Endocrinology, 2020, 16 (7): 395-400.

[4] KHARROUBI A T, DARWISH H M. Diabetes mellitus: The epidemic of the century [J]. World J Diabetes, 2015, 6(6): 850-67.

[5] IKEDA S, KOBAYASHI M, TAJIMA N. Cost-effectiveness analysis of voglibose for prevention of type 2 diabetes mellitus in Japanese patients with impaired glucose tolerance [J]. J Diabetes Investig, 2010, $1(6): 252-8$.

[6] GONG Q, ZHANG P, WANG J, et al. Changes in Mortality in People With IGT Before and After the Onset of Diabetes During the 23-Year Follow-up of the Da Qing Diabetes Prevention Study [J]. Diabetes care, 2016, $39(9): 1550-5$.

[7] ASSOCIATION A D. 3. Prevention or Delay of Type 2 Diabetes: Standards of Medical Care in Diabetes2019 [J]. Diabetes care, 2019, 42 (Supplement 1): S29.

[8] ZHANG J, XU J, HU X, et al. Diagnostic Method of Diabetes Based on Support Vector Machine and Tongue Images [J]. Biomed Research International, 2017, 1-9.

[9] LI J, YUAN P, HU X, et al. A tongue features fusion approach to predicting prediabetes and diabetes with machine learning [J]. Journal of Biomedical Informatics, 2021, 103693.

[10] JIATUO X, LIPING T, ZHIFENG $Z$, et al. The region partition of quality and coating for tongue image based on color image segmentation method; proceedings of the 2008 IEEE International Symposium on IT in Medicine and Education, F 12-14 Dec. 2008, 2008 [C]. 
[11] XU J, SUN Y, ZHANG Z, et al. Analysis and recognition of tongue texture features based on difference statistical method [J]. Journal of Shanghai University of Traditional Chinese Medicine, 2003, 17 (3): 558.

[12] EKOE J-M, GOLDENBERG R, KATZ P. Screening for Diabetes in Adults [J]. Canadian Journal of Diabetes, 2018, 42 (S16-S9.

[13] KHAN T, TSIPAS S, WOZNIAK G. Medical Care Expenditures for Individuals with Prediabetes: The Potential Cost Savings in Reducing the Risk of Developing Diabetes [J]. Popul Health Manag, 2017, 20(5): 389-96.

[14] ROWLEY W R, BEZOLD C, ARIKAN Y, et al. Diabetes 2030: Insights from Yesterday, Today, and Future Trends [J]. Popul Health Manag, 2017, 20(1): 6-12.

[15] KAUHL B, SCHWEIKART J, KRAFFT T, et al. Do the risk factors for type 2 diabetes mellitus vary by location? A spatial analysis of health insurance claims in Northeastern Germany using kernel density estimation and geographically weighted regression [J]. Int J Health Geogr, 2016, 15(1): 38-.

[16] HSU C-C, LEE C-H, WAHLQVIST M L, et al. Poverty increases type 2 diabetes incidence and inequality of care despite universal health coverage [J]. Diabetes care, 2012, 35(11): 2286-92.

[17] HSU P-C, WU H-K, HUANG Y-C, et al. The tongue features associated with type 2 diabetes mellitus [J]. Medicine, 2019, 98(19): e15567-e. 
Figures

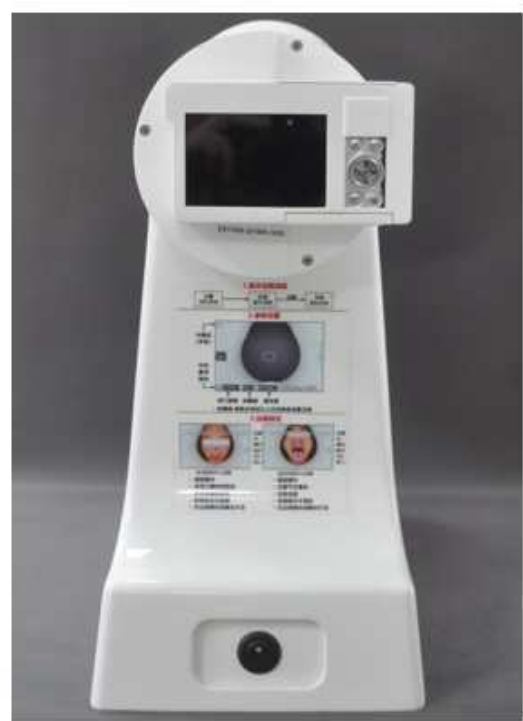

(a) Rear view

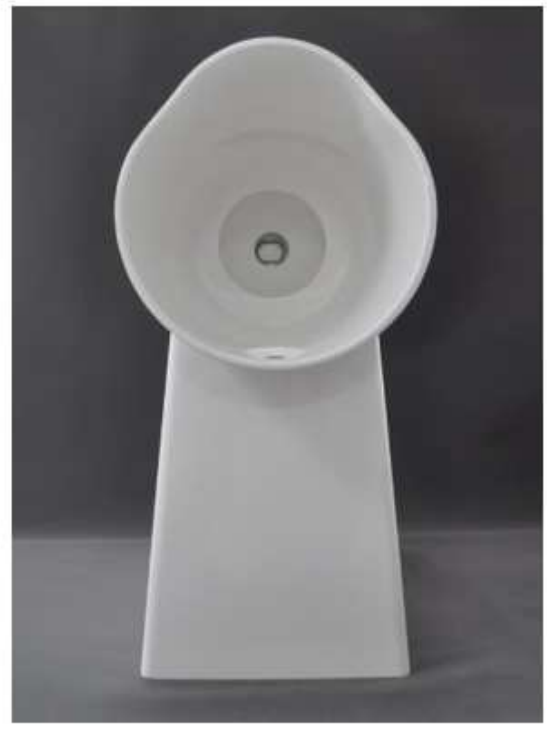

(b) Front view

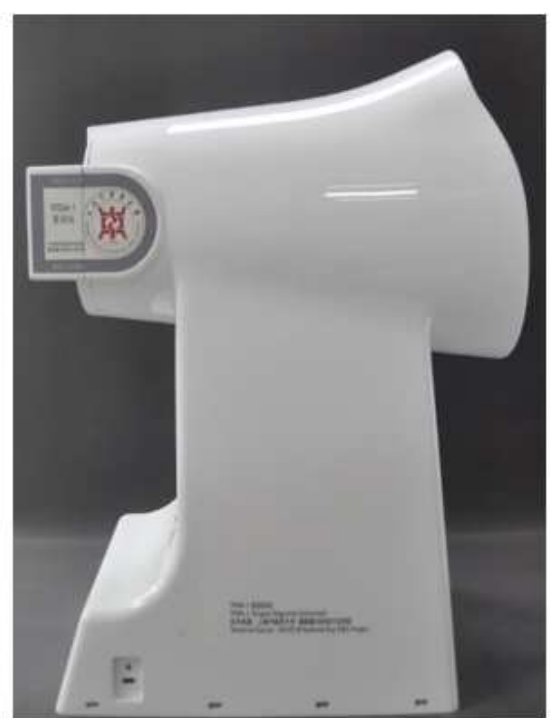

(c) Side view

\section{Figure 1}

Type TFDA-1 tongue diagnosis instrument

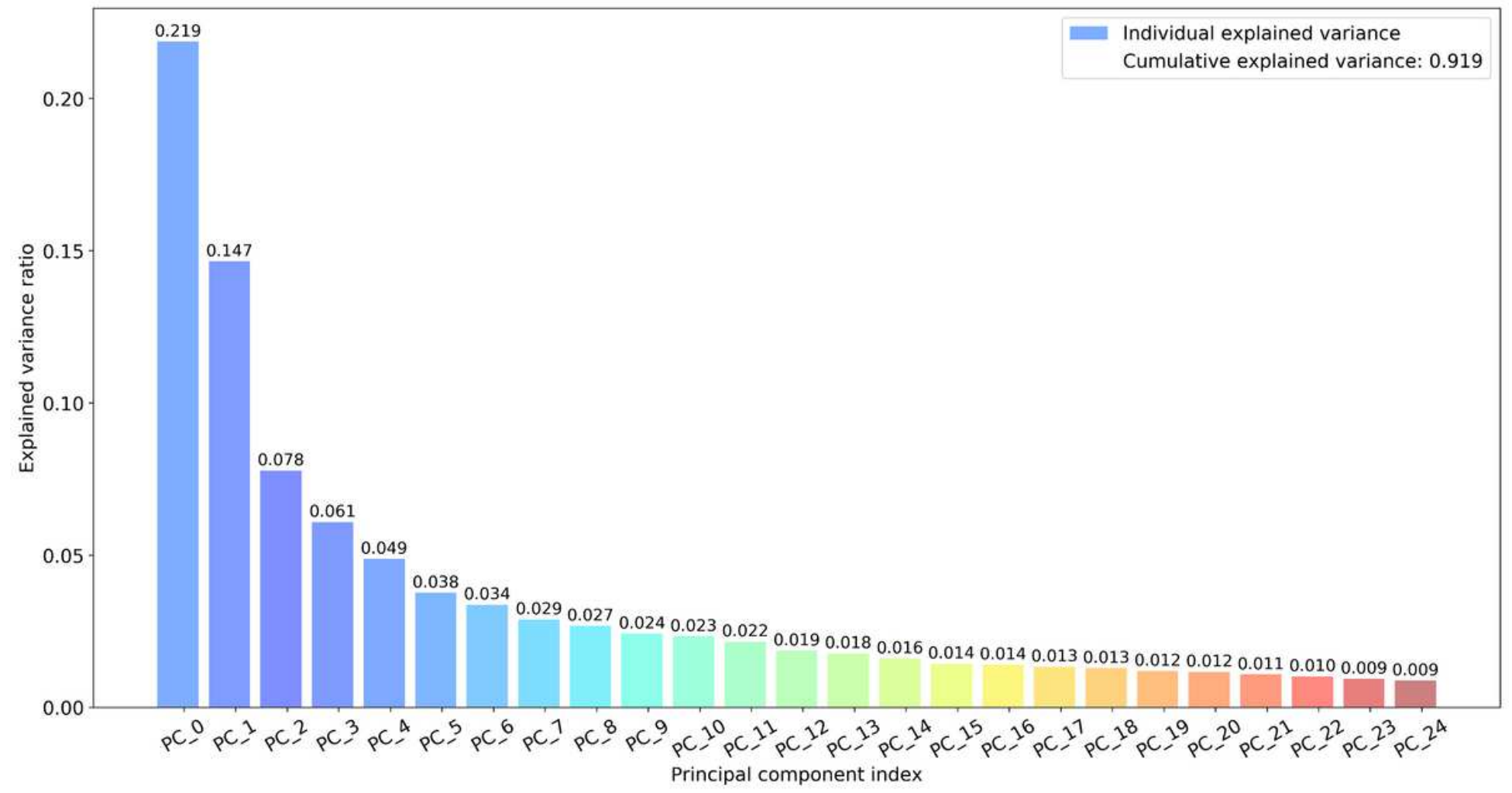

Figure 2

Proportion of variance explained by PCs in prediabetes group 


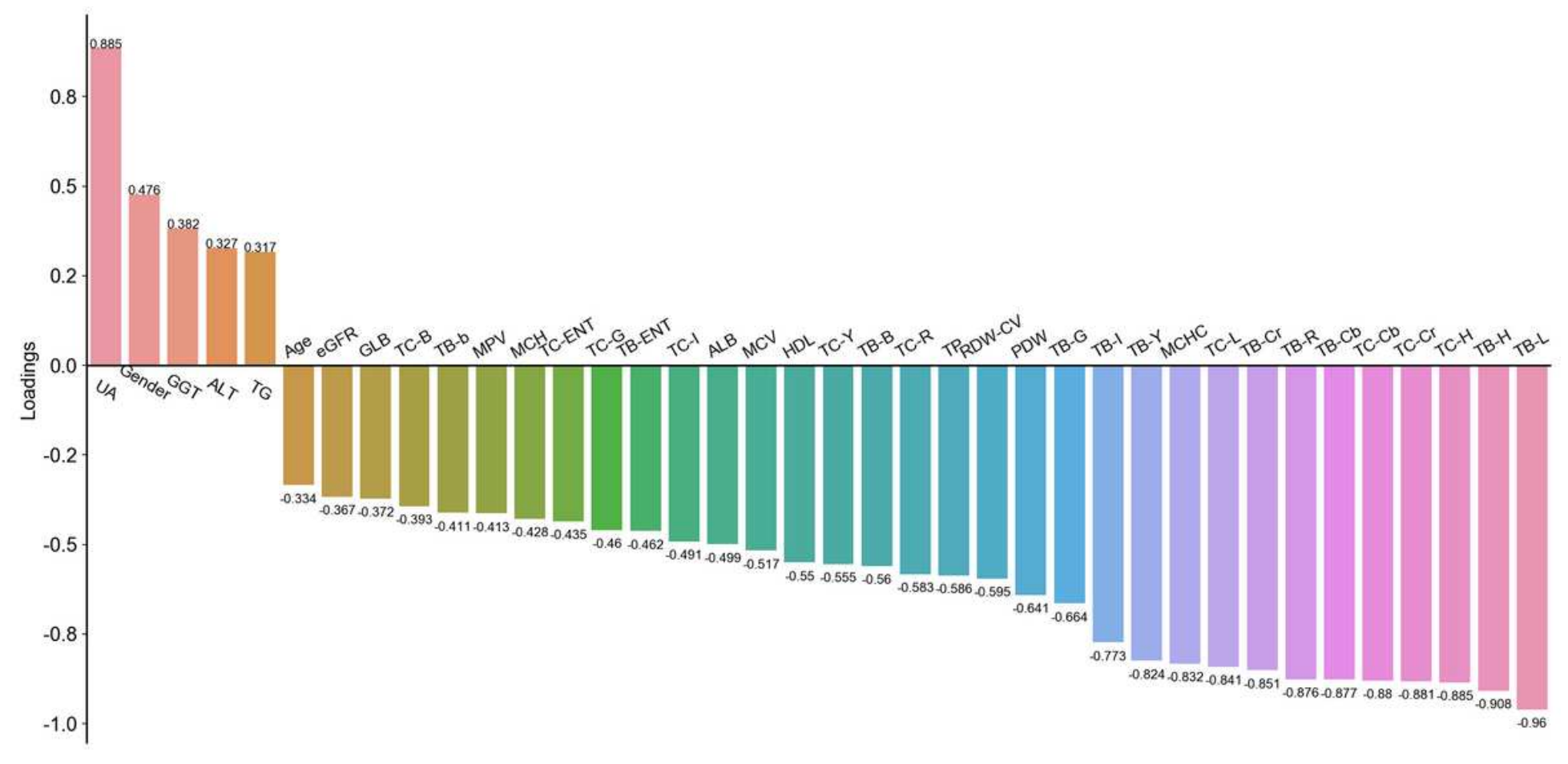

Figure 3

Factor loadings of PC_0 in prediabetes group Note: TB-Cr, TB-ENT, TC-ENT are negatively correlated with the risk factor PC_0, and the correlations are $-0.851,-0.462$, and -0.435 , respectively.

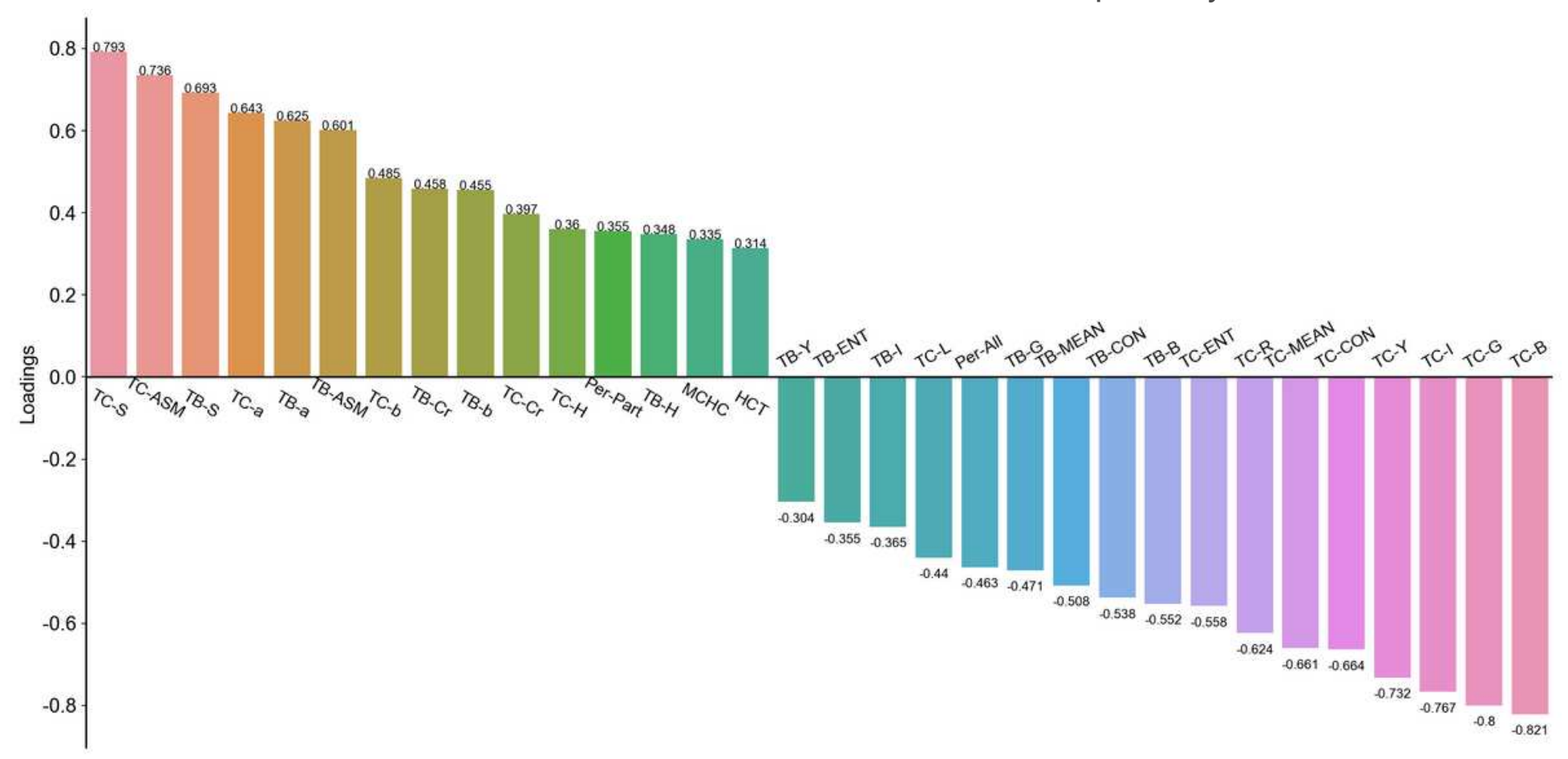

\section{Figure 4}

Factor loadings of PC_1 in prediabetes group Note: PC_1 is a protective factor, TC-ASM, TB-ASM, TB-Cr and PC_1 are positively correlated, and the correlation coefficients are $0.736,0.601$ and 0.458 , 
respectively. TC-CON, TC-MEAN, TC-ENT, TB-CON, TB-MEAN, TB-ENT are negatively correlated with PC_1, the correlation coefficients are $-0.664,-0.661,-0.558,-0.538,-0.508,-0.355$, respectively.

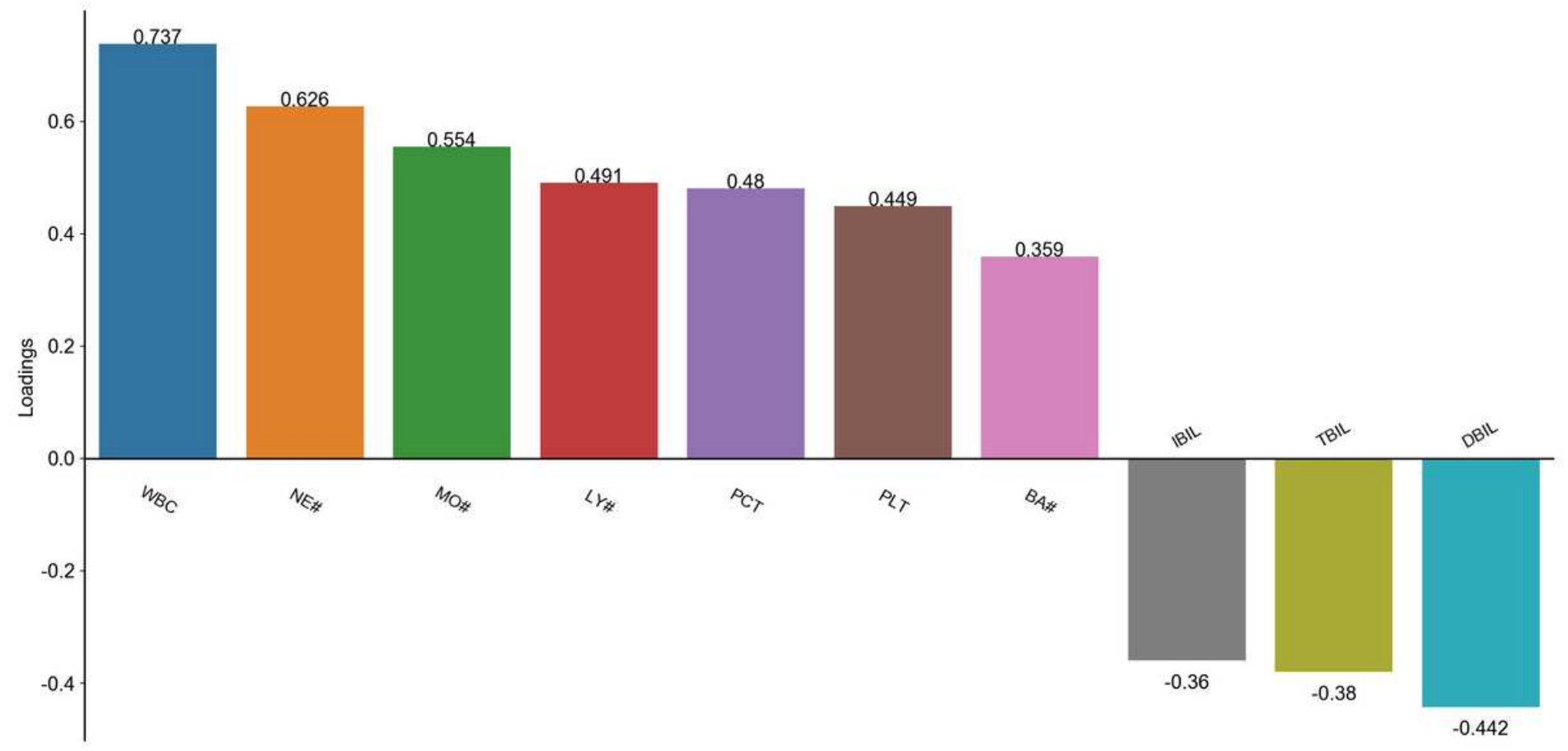

Figure 5

Factor loadings of PC_4 in prediabetes group Note: PC_4 is a risk factor, WBC, NE\#, LY\# are positively correlated with PC_4, and the correlation coefficients are $0.737,0.626,0.491$, respectively. 


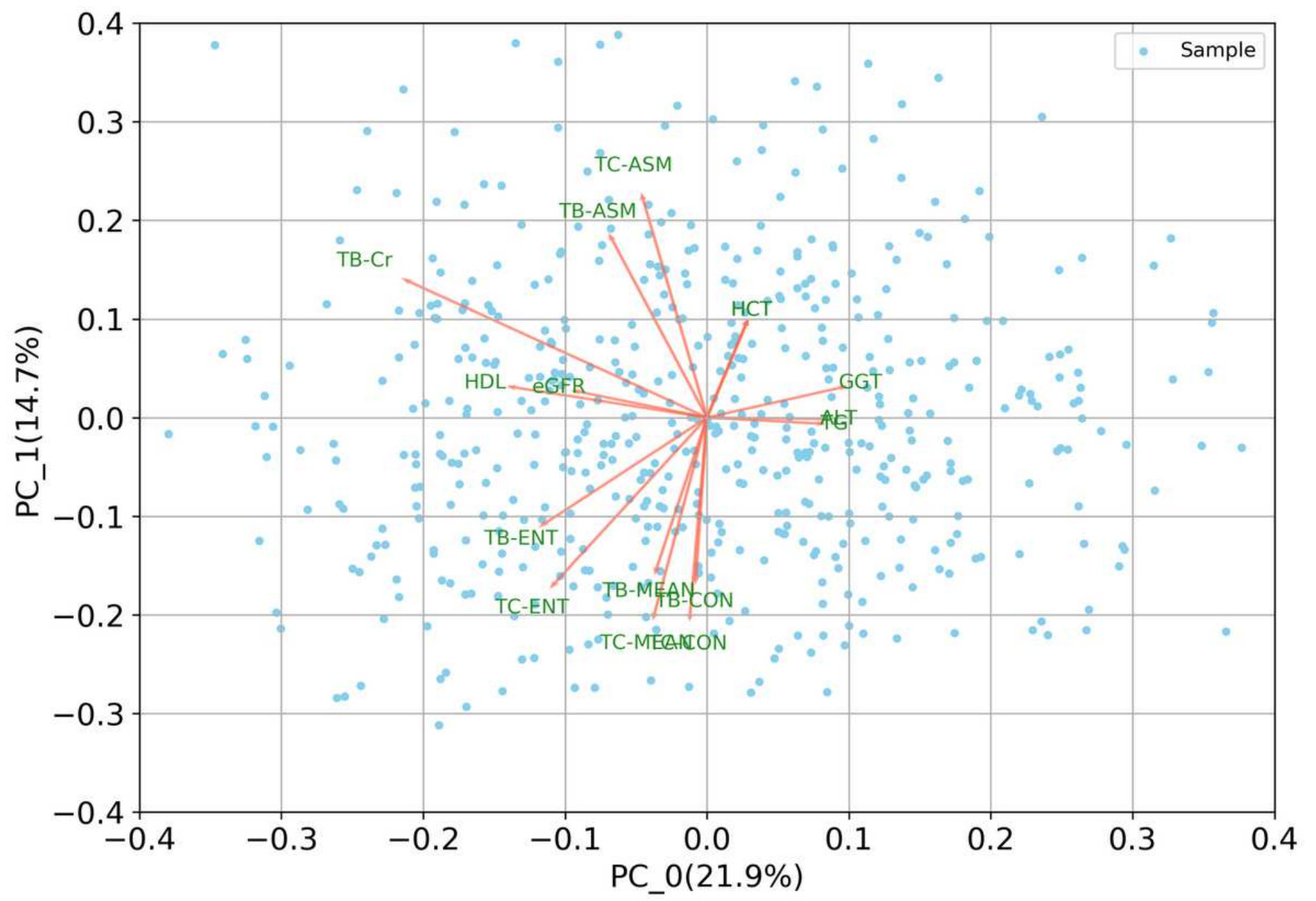

Figure 6

Biplot of PC_0 and PC_1 in prediabetes group Note: A biplot shows how strongly each feature influences a principal component. The position coordinates of the feature are determined by the weight of feature of the corresponding PC. The angle between features indicates the correlation between features. An acute angle $(<900)$ indicates a positive correlation between two features. Right angles (900) indicate irrelevance. Obtuse angles ( $>900$ ) indicate negative correlation. PC_0 is a risk factor, TB-Cr mainly acts on PC_0 (The vector that is sub-parallel to the $x$-axis), and TB-Cr has a reverse effect on PC_0 and is a protective factor. PC_1 is a protective factor, TB-ASM, TC-ASM, TB-CON, TC-CON, TB-MEAN, TC-MEAN, TCENT mainly act on PC_1 (The vectors that are sub-parallel to the $y$-axis). TB-ASM and TC-ASM play a positive role on PC_1 and are protective factors; TB-CON, TC-CON, TB-MEAN, TC-MEAN and TC-ENT play a negative role on PC_1 and are risk factors. 


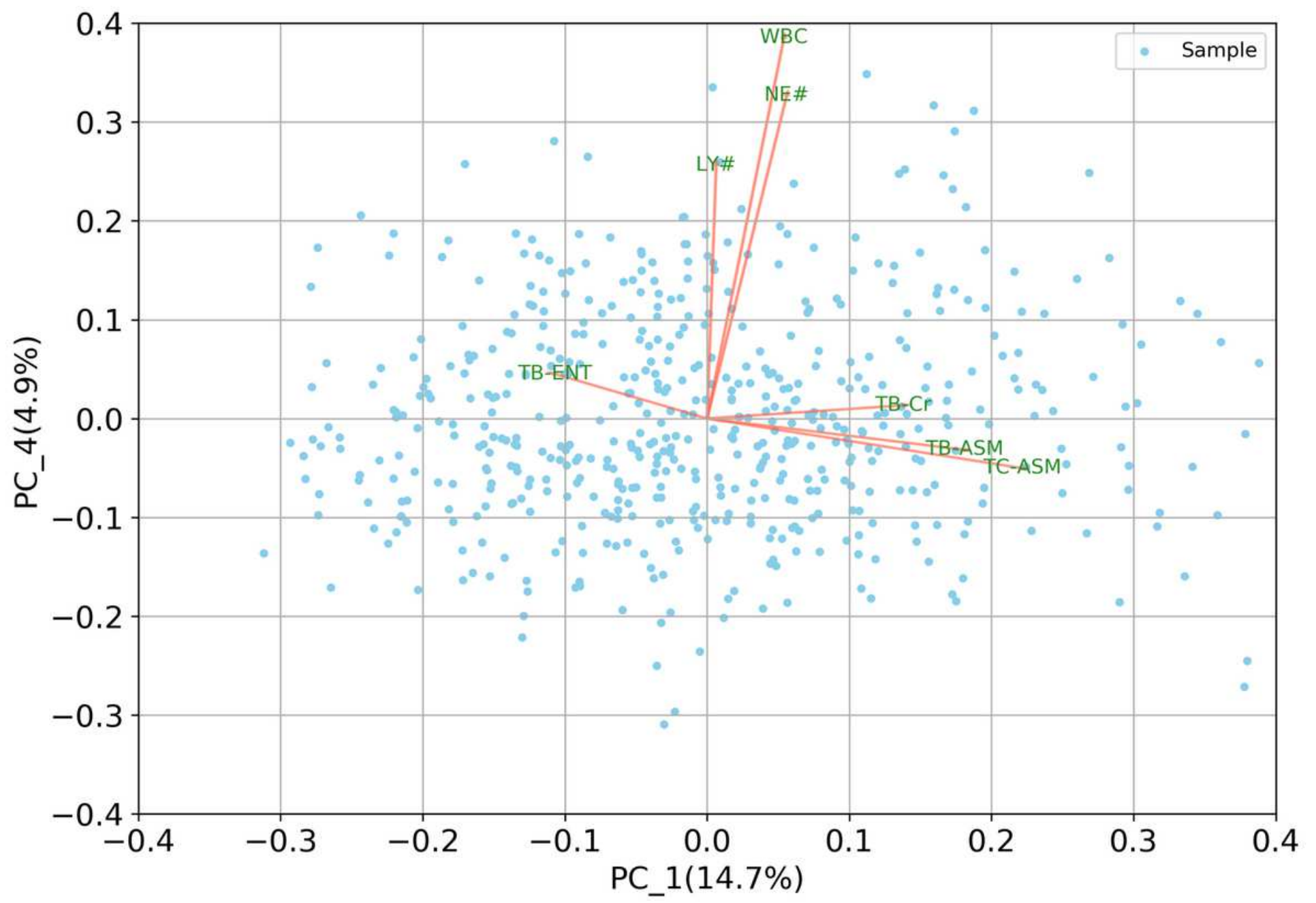

Figure 7

Biplot of PC_1 and PC_4 in prediabetes group Note: PC_1 is a protective factor, TB-ENT mainly act on PC_1 (The vector that is sub-parallel to the $x$-axis). TB-ENT plays a negative role on PC_1 and is risk factor. 


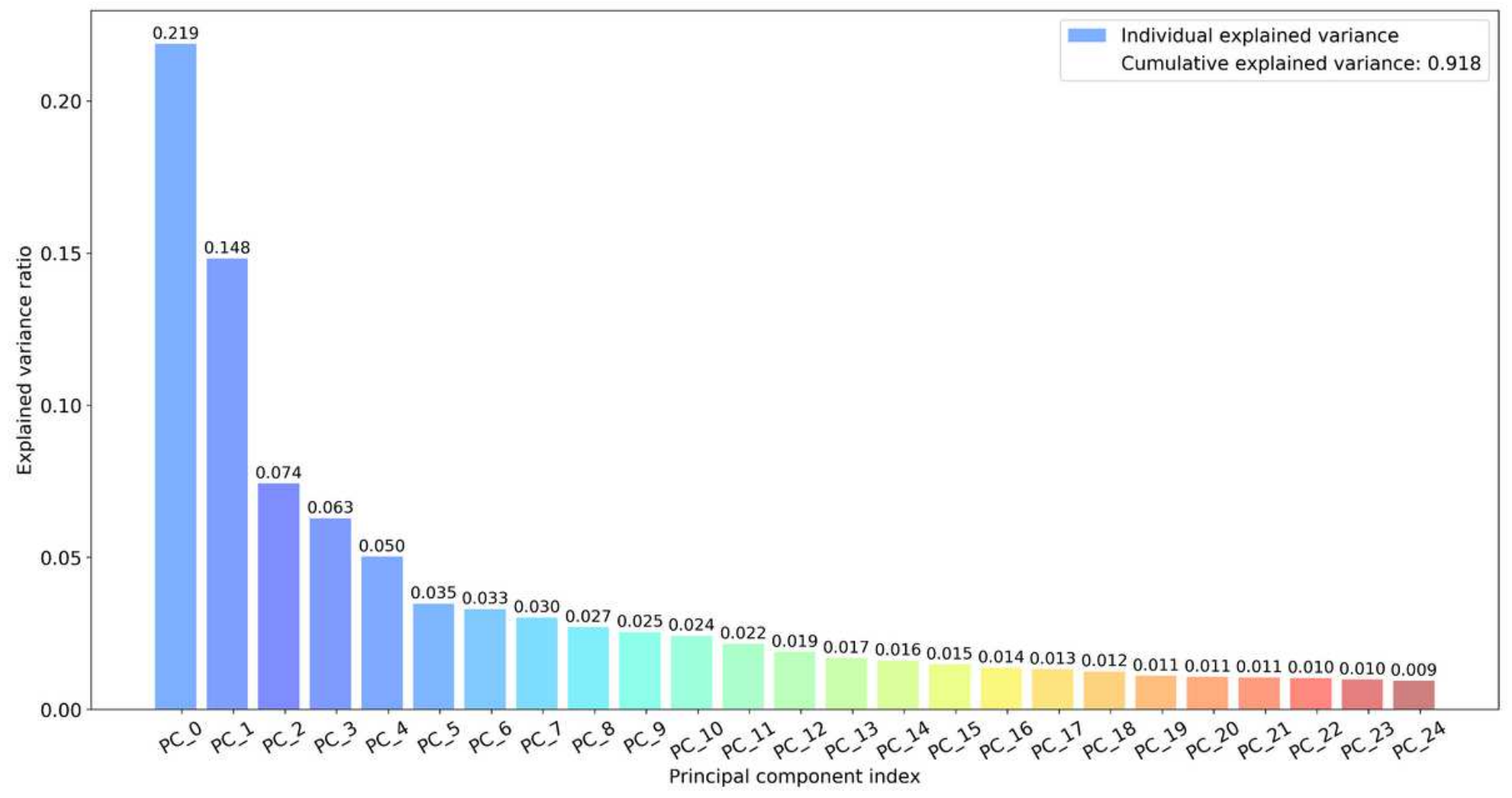

Figure 8

Proportion of variance explained by PCs in diabetes group

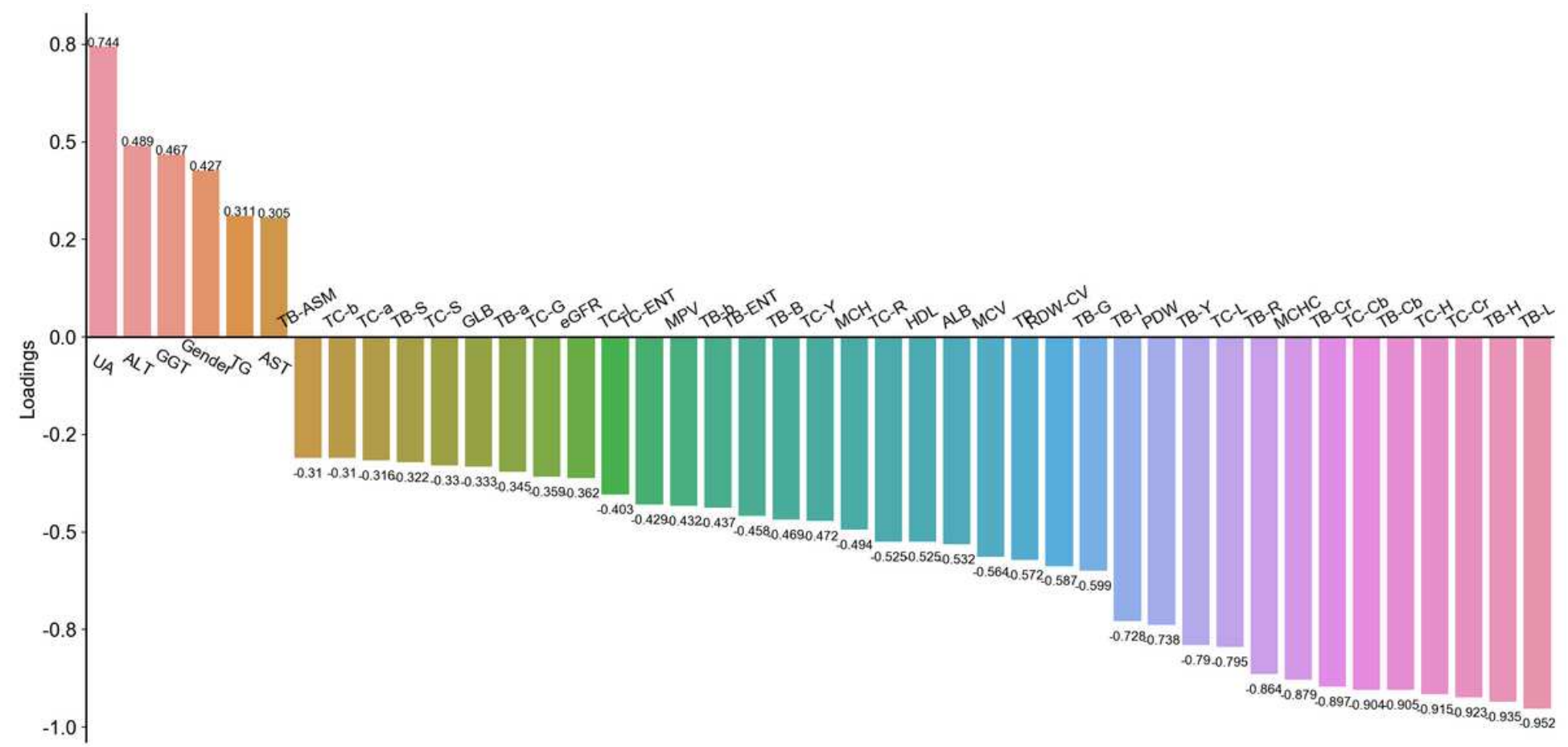

Figure 9

Factor loadings of PC_0 in diabetes group Note: PC_0 is a risk factor, TB-Cb, TB-Cr, TB-b, TB-a, TB-S, TC-b are negatively correlated with PC_0, and the correlation coefficients are $-0.905,-0.897,-0.437,-0.345$, 
$-0.322,-0.31$, respectively.

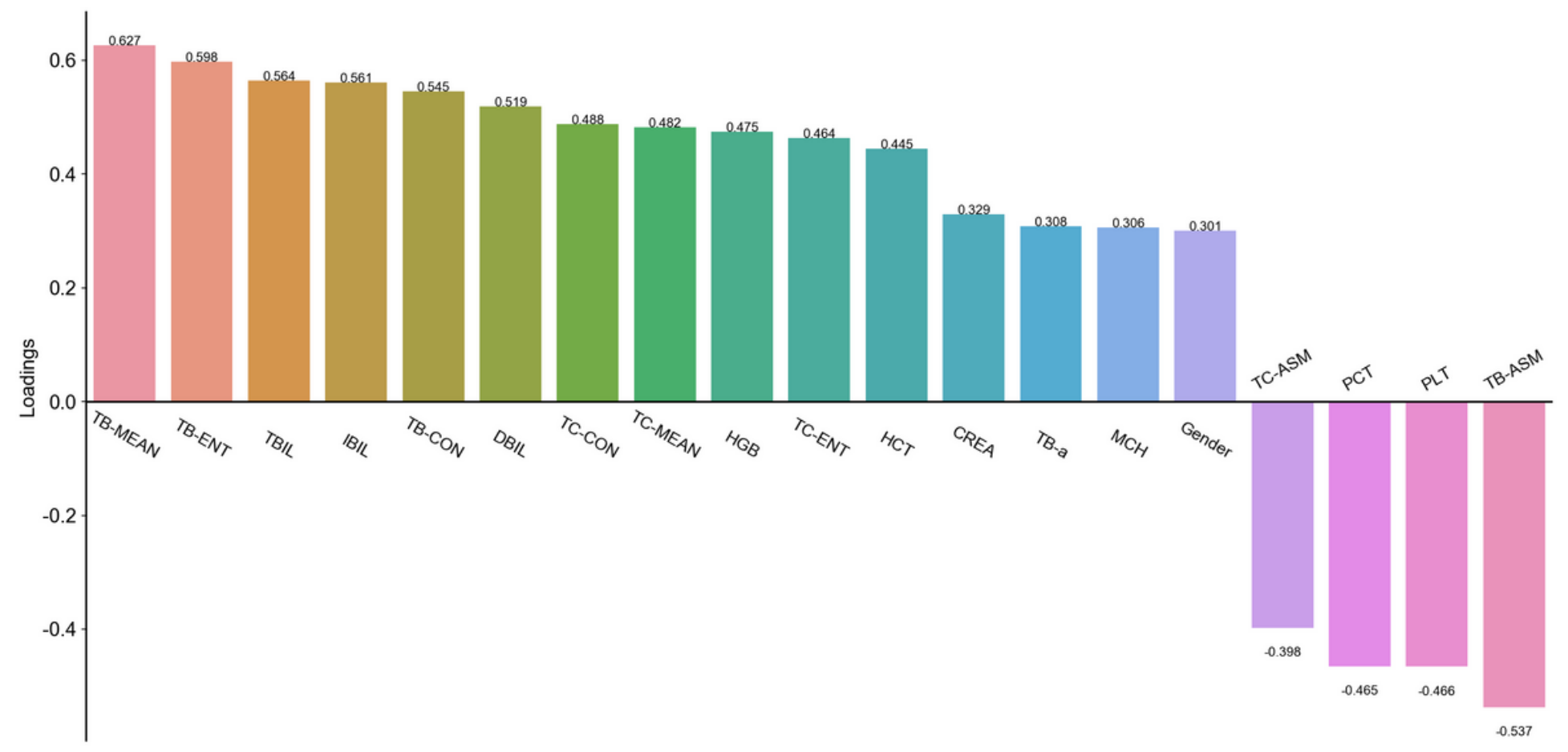

Figure 10

Factor loadings of PC_2 in diabetes group Note: PC_2 is a protective factor, TB-a is positively correlated with PC_2, and the correlation coefficient is 0.308 .

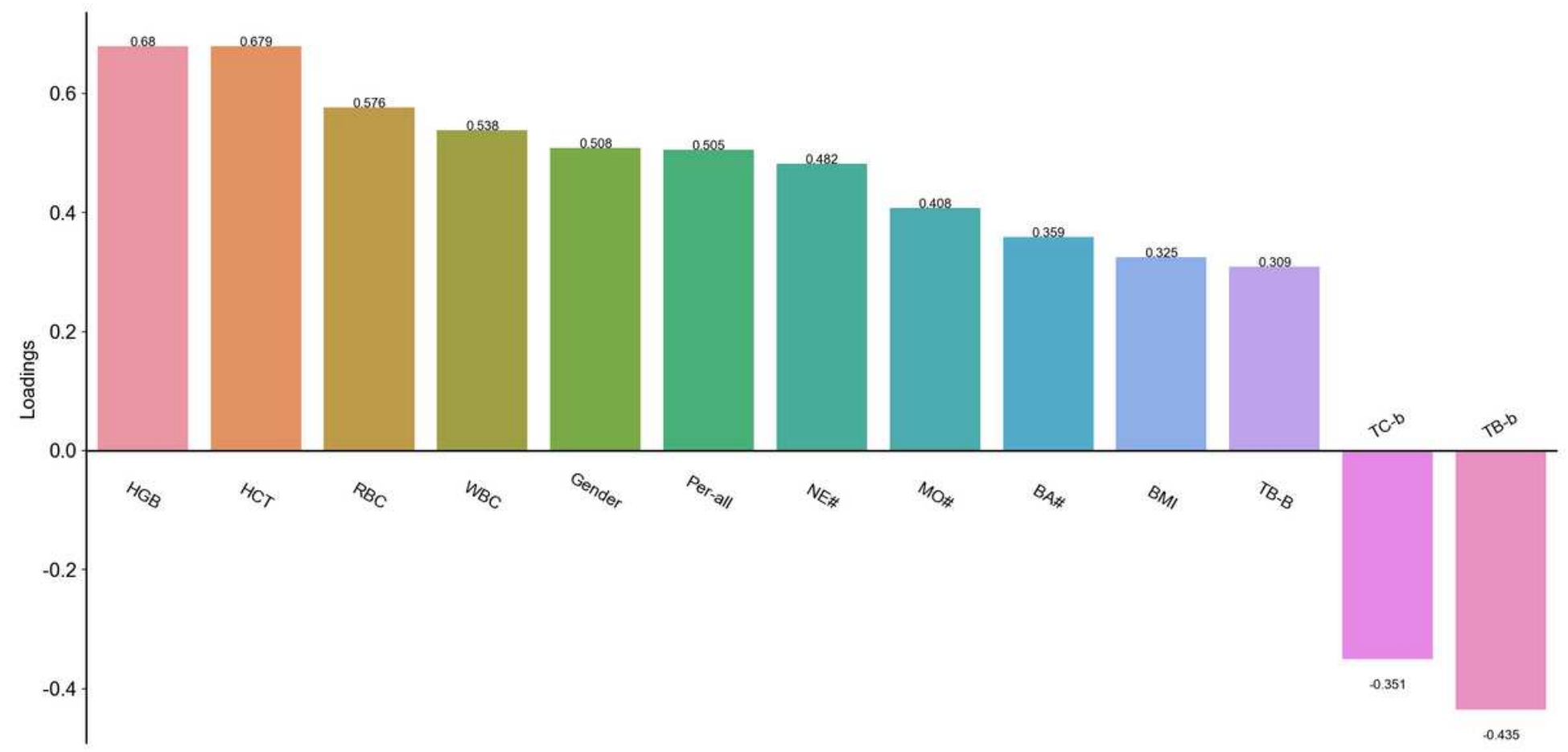

Figure 11 
Factor loadings of PC_3 in diabetes group Note: PC_3 is a risk factor, TB-b and TC-b are negatively correlated with PC_3, and the correlation coefficients are -0.435 and -0.351 , respectively.

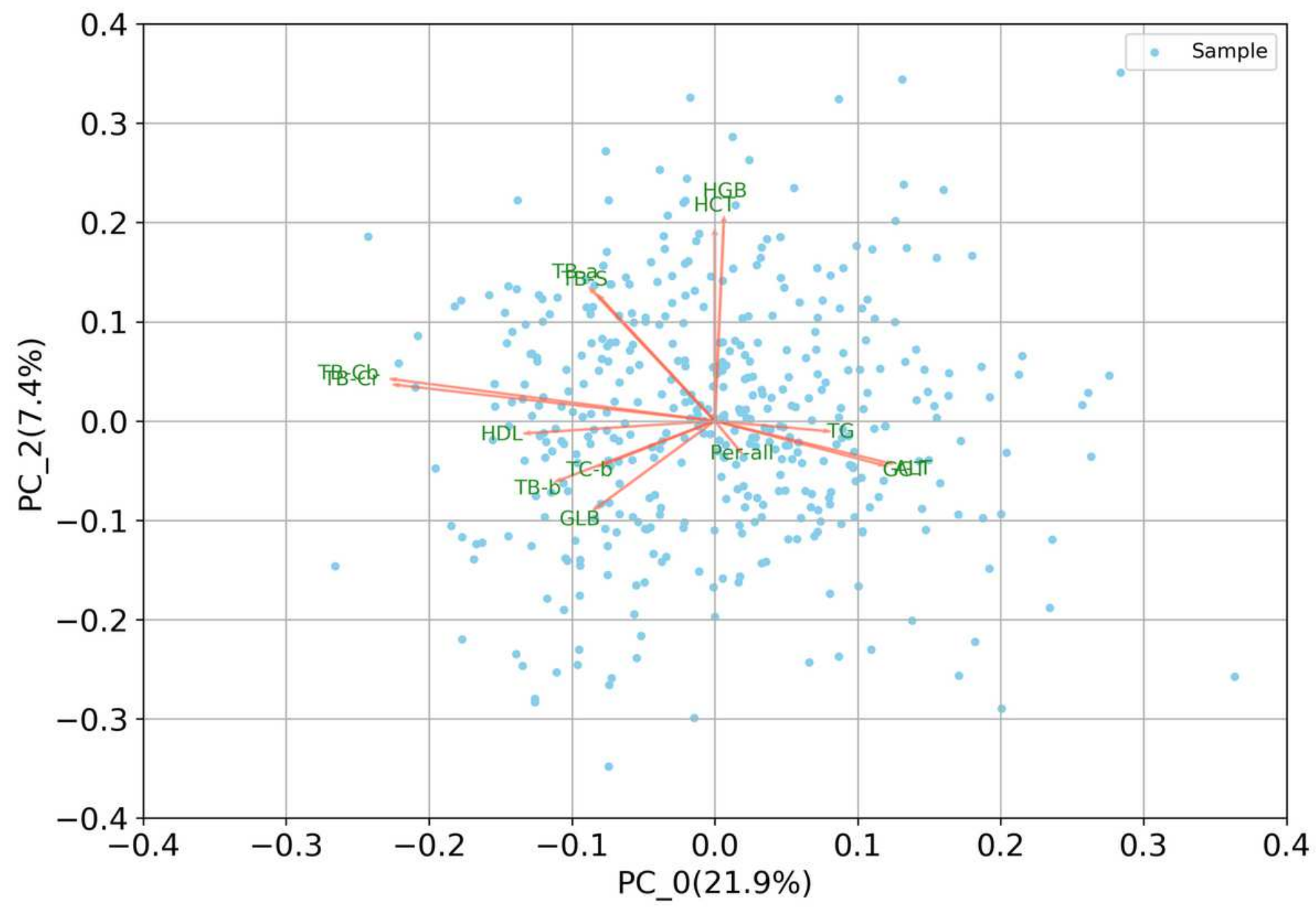

Figure 12

Biplot of PC_0 and PC_2 in diabetes group Note: PC_0 is a risk factor, TB-Cr, TB-b, TC-b mainly act on PC_0 (The vectors that are sub-parallel to the $x$-axis), TB-Cr, TB-b and TC-b play a negative role on PC_0 and are protective factors. PC_2 is a protective factor, TB-a and TB-S mainly act on PC_2 (The vectors that are sub-parallel to the $y$-axis), TB-a and TB-S positively act on PC_2 as a protective factor. 


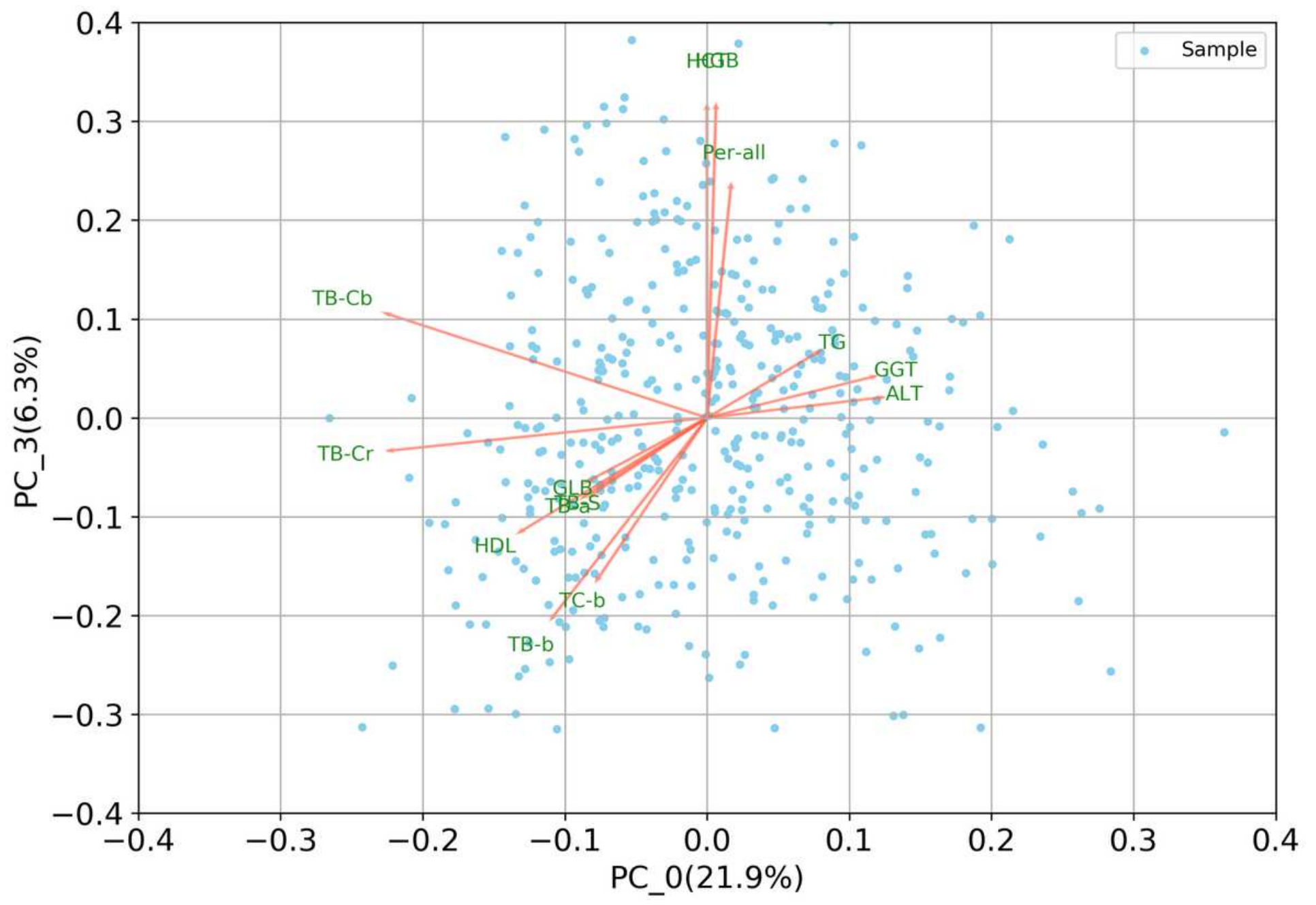

Figure 13

Biplot of PC_0 and PC_3 in diabetes group Note: PC_3 is a risk factor, Per-all mainly acts on PC_3 (The vector that is sub-parallel to the $y$-axis), Per-all has a positive effect on PC_3 and is a risk factor. 


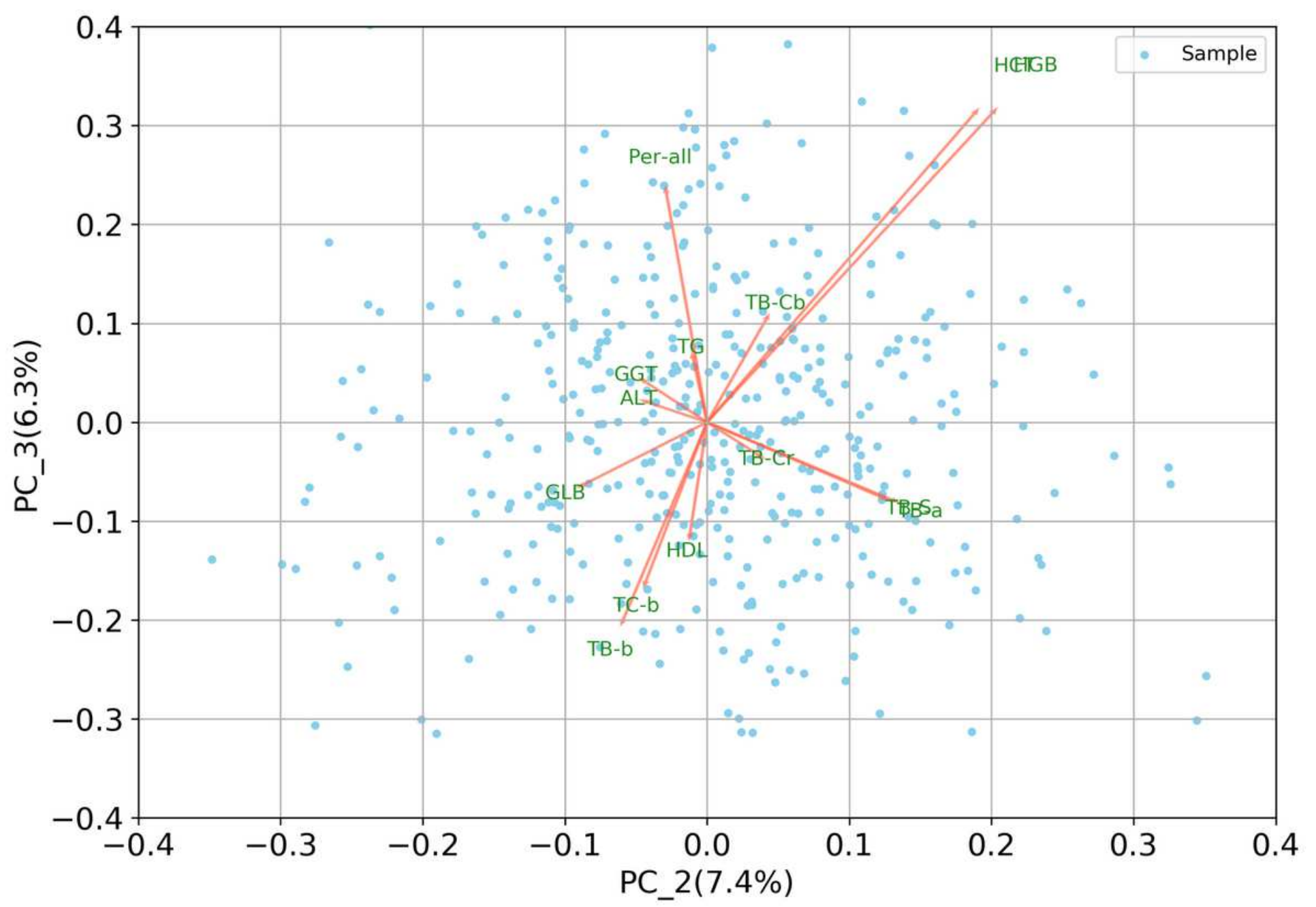

Figure 14

14 Biplot of PC_2 and PC_3 in diabetes group Note: PC_3 is a risk factor, TB-Cb mainly acts on PC_3 (The vector that is sub-parallel to the $y$-axis), TB-Cb has a positive effect on PC_3 and is a risk factor. 